University of Wollongong

Research Online

SMART Infrastructure Facility - Papers

Faculty of Engineering and Information

Sciences

$1-1-2015$

Pathway variation analysis (PVA): modelling and simulations

Nagesh Shukla

University of Wollongong, nshukla@uow.edu.au

Sudi Lahiri

University of Warwick

Darek Ceglarek

University of Warwick

Follow this and additional works at: https://ro.uow.edu.au/smartpapers

Part of the Engineering Commons, and the Physical Sciences and Mathematics Commons

Research Online is the open access institutional repository for the University of Wollongong. For further information contact the UOW Library: research-pubs@uow.edu.au 


\title{
Pathway variation analysis (PVA): modelling and simulations
}

\begin{abstract}
Maintaining a care pathway within a hospital to provide complex care to patients is associated with challenges related to variations from the pathway. This occurs due to ineffective decision-making processes, unclear process steps, the interactions, conflicting performance measures for speciality units, and the availability of resources. These variations from the care pathway or standard care delivery processes lead to longer patient waiting times and lower patient throughput. Traditional approaches to improve the pathway focus primarily on reducing variations within the care pathway such as bottlenecks or throughput within the pathway rather than examining variations from the care pathway. In this study, we propose a novel methodology, called pathway variation analysis (PVA), to identify, simulate and analyse variations from the patient care pathways. PVA method includes patient ward level journey dataset and qualitative staff interviews to simulate patient variations. The proposed methodology had been applied to the stroke care services of a hospital, which increased their key performance from $73 \%$ to $84.97 \%$. A PVA methodology is proposed which simulated patient diversions from the care pathway by modelling hospital operational parameters, assessing the accuracy of clinical decisions and performance measures of speciality units involved. The proposed methodology can be applied to other care pathways settings to reduce patient diversion from the care pathway.
\end{abstract}

\section{Keywords}

variation, simulations, pathway, modelling, pva, analysis

Disciplines

Engineering | Physical Sciences and Mathematics

\section{Publication Details}

Shukla, N., Lahiri, S. \& Ceglarek, D. (2015). Pathway variation analysis (PVA): modelling and simulations. Operations Research for Health Care, 6 61-77. 


\title{
Pathway variation analysis (PVA): Modelling and Simulations
}

\author{
Nagesh Shukla $^{1,2}$, Sudi Lahiri ${ }^{2}$, Darek Ceglarek ${ }^{2,3}$ \\ ${ }^{1}$ SMART Infrastructure Facility, University of Wollongong, Wollongong, 2522, Australia \\ 2 The Digital Laboratory, WMG, University of Warwick, Coventry, CV4 7AL, UK \\ ${ }^{3}$ Department of Industrial \& Systems Engineering, University of Wisconsin, Madison, WI 53706, USA
}

Corresponding Author:

Dr. Nagesh Shukla,

SMART Infrastructure Facility, University of Wollongong, Wollongong, 2522, Australia

Tel: +61 242392329

Fax: +61 242211489

Email: nshukla@uow.edu.au 


\section{Abstract:}

Maintaining a care pathway within a hospital to provide complex care to patients is associated with challenges related to variations from the pathway. This occurs due to ineffective decision-making processes, unclear process steps, the interactions, conflicting performance measures for speciality units, and the availability of resources. These variations from the care pathway or standard care delivery processes lead to longer patient waiting times and lower patient throughput. Traditional approaches to improve the pathway focus primarily on reducing variations within the care pathway such as bottlenecks or throughput within the pathway rather than examining variations from the care pathway. In this study, we propose a novel methodology, called pathway variation analysis (PVA), to identify, simulate and analyse variations from the patient care pathways. PVA method includes patient ward level journey dataset and qualitative staff interviews to simulate patient variations. The proposed methodology had been applied to the stroke care services of a hospital, which increased their key performance from $73 \%$ to $84.97 \%$. A PVA methodology is proposed which simulated patient diversions from the care pathway by modelling hospital operational parameters, assessing the accuracy of clinical decisions and performance measures of speciality units involved. The proposed methodology can be applied to other care pathways settings to reduce patient diversion from the care pathway.

\section{Introduction}

Variations have been identified as a major challenge in healthcare systems [1-4]. These variations are often linked with the medical errors in care delivery [5]. Weingart, et al., [6] estimated that medical error results in 44000-98000 unnecessary deaths each year in the United States. Reducing care variations by standardizing the care delivery processes or developing and implementing an integrated care pathway (ICP) has been identified as an effective approach [7-12].

An integrated care pathway (ICP) is a set of guidelines or care which is to be provided to patients with a particular presenting condition [13]. ICP generally involves multidisciplinary communication among several speciality units in the hospital to efficiently provide care to the patients. ICPs are implemented in hospitals to reduce and control care delivery variations [14], however, effectively implementing ICPs is often associated with problems due to large variations from the set care pathway [14][46]. These variations lead to reduced performance of daily care [46]. For example, placing patients, requiring specialist treatment, in non-speciality units due to unclear processes can compromise the patient care delivered [48]. This means that the causes for such variations need to be understood.

Research into the identification of variations from care pathways and pathway improvements is limited. The majority of research studies are focused on developing and implementing integrated care pathways rather than identifying variations from the pathways when these are implemented [11] [13] [18-20]. Several methods involved in improving care pathways by reducing variations have been 
identified in the literature [17][25][30]. These can be broadly classified into: (i) process redesign, (ii) diagnostic accuracy and (iii) efficiency improvements or optimum resource utilisation.

Methods related to process redesign have been well researched [17] [21-23]. Process redesign generally involves a review of the current processes by developing process model for suggesting improvements.

The process model describes the flow of information, patients and staff, the service delivery decisions, and the essential inter-relationships and interdependence among the process steps. Several studies have employed models such as flowcharts [21], data flow diagrams [32], integrated definition for function modelling (IDEF, [22]), value stream mapping [15][33], and role activity diagrams [1617][34] and these are used to examine the service delivery process, to analyse clinical information systems, and information system requirements. Shukla et al. [17] have proposed that RAD is suitable for service delivery in healthcare. However, process modelling and redesign alone will not be sufficient to achieve overall improvement in care pathways. This is due to the involvement of several disease-specific clinical decisions and operational constraints specific to the individual hospital.

Improving diagnostic accuracy by improving clinical tests to categorise patients accurately and treat them appropriately is another method. Several studies have been conducted by designing and assessing clinical tests to improve diagnostic accuracy [24-27]. Some of the clinical tests involved in complex care pathways such as stroke are FAST (Face Arm Speech Test, [24], ROSIER (Recognition of Stroke in the Emergency Room [25]), NIHSS (National Institute of Health Stroke Scale [26]), OCSP (Oxfordshire Community Stroke Project clinical classification [27]), CT/MRI scans [35-36]. These clinical tests have been developed to reduce delays in accurately identifying the relevant subgroup of patients for treatment using the care pathway. In general, however, care pathways involve multiple clinical tests and hospital processes which are linked with each other. Hence, overall improvement within care pathways requires an integrated approach.

Another method in improving care pathways is due to the need to ensure optimum utilization of resources. These methods have been examined in previous research studies to develop a prototype model for resource planning, bottleneck analysis, and exploring alternatives to improve care delivery [28-31]. This involves simulation modelling of care delivery for performance improvement. Research has been conducted to simulate hospital operations for optimum resource utilization, to reduce waiting times and length of stay (LOS), to increase throughput of the care delivery processes [28][37-39]. Simulation modelling has been applied to various healthcare areas such as a vascular surgery [29], accident and emergency [30], intermediate care [31] and the evaluation of screening programmes [4041]. Shukla et al. [50] have used modelling for the accessibility based radiotherapy facility location for cancer patients. Sangi et al. [51] have used various data mining methods for assessing the diabetes complications. The majority of these simulation models have been developed based on inbuilt flow diagrams available in most of the commercial discrete even simulation (DES) software packages. The initial flow diagrams used in commercial DES are not suitable to represent the complexity of care 
pathways [47]. This is partly due to the fact that flow diagrams are best suited to representing sequential processes rather than representing the complex integrated and interacting processes common in care pathways [17]. This means that the use of simulation models alone in complex care scenarios is not sufficient.

Each of abovementioned approaches has certain advantages but there is a lack of interdisciplinary approaches which could integrate different methods to analyse variations from the care pathway. To do this, a pathway variation analysis (PVA) methodology was proposed. The proposed methodology involves role activity diagrams (RAD) to model the detailed care pathways. These system-level process models were then used together with hospital IT system data to identify critical decisions in the care pathway which can lead to patient diversion. The data used in this research mainly include patient's ward level journey data and time durations for various process steps (see supplementary material for detailed list of data variables used). The critical decisions were simulated and analysed together with operational parameters and performance measures of the speciality units involved in the care pathway. As a result of this analysis, set-based solutions were generated to reduce unnecessary variations from the care pathway.

\section{Pathway Variations Modelling \& Simulation Methodology}

In this section, variations modelling \& simulation methodology is described to identify variations from care pathways for service improvements. Overall methodology consists of few major steps as illustrated in Fig. 1.

In Fig. 1, proposed approach starts with system-level modelling of care pathway of the hospital. The care pathway modelling involves representation of processes involved within a particular care pathway such as activities, interactions, roles, and decisions. Once developed, problem with care pathway of a hospital is identified. The problem identification step generally identifies two types of problem: (i) low patient throughput, and/or (ii) patient diversions from care pathway. This paper mainly focuses on patient diversions; therefore, analysis methodology for low throughput problem is not discussed. Pathway variations analysis method is then used to identify critical decisions which significantly lead to patient diversions. These decisions are then modelled to reduce patient diversions from the pathway. Following subsections details each of the steps of the methodology.

\subsection{System-level modelling of healthcare service delivery}

In this sub-section, system level modelling of healthcare service delivery process is discussed. The system-level modelling is defined in this step as two sub-step procedure: (i) integrated care pathway modelling in a hospital; and (ii) clinical decision making process modelling. These steps require process mapping or modelling to identify and represent complex collaborative service delivery processes followed in hospital. Care pathway and clinical decision making process models helps to understand interactions among medical speciality units (including accident \& emergency (A\&E)), 
roles involved in providing care, and decision making steps. Details about each of the sub-steps are provided as follows:

\subsubsection{Care pathway modelling}

The care pathway modelling employs role activity diagram (RAD) for modelling details about complex care pathway in hospital. RAD based modelling methodology uses interviews of key staffs, involved within care pathway, for representing processes within care pathway followed in hospital (see [17] for more details on methodology for RAD development).

\subsubsection{Decision making process modelling}

In this sub-step, decision making processes which are crucial in delivery of care to patients within pathway are modelled. The modelling of crucial decision making processes helps to analyse variations from care pathway implemented in hospital. In this step, decision maker(s), interactions, information sources are mapped. More information about decision making modelling is provided in the supplementary material.

\subsection{Pathway variation analysis (PVA)}

The pathway variations such as patient diverted to non-speciality wards are largely due to the ineffective push and pull factors among multiple specialty units involved within a care pathway. The push factor for A\&E is defined as - sending patients off to speciality unit (within the care pathway) regardless of unit's capacity to handle incoming patient. For e.g., push factors include A\&E 4-hours target. On the other hand, pull factor is the speciality unit's decision to take new patients based on its current capacity. For e.g. number of free ward beds is used to make decision on admitting new patients. Figure 2 illustrates patient movements from one speciality ward to another starting from A\&E. In Fig. 2, patients can be transferred from one speciality unit to another unit in the care pathway or patients can be discharged. However, due to the mismatch of push and pull factors among speciality units in the care pathway, patients are diverted from the care pathway. The patient diversions from care pathway to other medical units compromises the care delivered to patients.

Pathway variations or variations from care pathway are used interchangeably in the rest of the paper. The main steps of PVA are - (i) generate a sample of patients for analysis, (ii) simulate patient flow on pathway and (iii) simulation analysis and suggestions. The details of the steps involved in PVA are discussed as follows:

\subsubsection{Generate sample of patients}

In this step, a pool or a sample of patients are virtually generated for analysis in next sub-section. The sample of patients in created based on the historical patient arrival times, and presenting conditions.

A. Sample Size: Sample size is crucial parameter in generating sample of patients for analysis. Let us consider $i^{t h}$ patient of the sample such that $i \in\left(1,2, \ldots, N_{p t s}\right)$, where $N_{p t s}$ is the total 
number of patients (or sample size of the sample of patients to be generated) coming to A\&E in a time period $T$ (in days, for e.g. week, or month, or year).

B. Patient Arrival Time: The patient arrival time at A\&E plays a major role in provision of care to patients as $A \& E$ is identified to be the crucial entry point for patients on pathway. Therefore, this model considers $i^{\text {th }}$ patient arrival time as $\boldsymbol{P} \boldsymbol{t}_{\text {arrival }}(i) \in[00: 00,23: 59]$ and has particular distribution. This helps in modelling patient arrival during a particular day.

C. Patient presenting conditions: Particular care pathway in hospital is used for treatment of a particular type of disease such as stroke, diabetes. Patients coming to A\&E for treatments can be broadly classified into disease which is treated in care pathway and disease mimics. Disease mimics are the patient conditions which mimics symptoms of disease treated in care pathway but are different and thus are not suitable for care provided in disease care pathway. Disease mimic patients coming to A\&E affect pathway variations as care providers in $A \& E$ can put patients on disease care pathway, which compromises the care provided to patients and lowers the productivity of care pathway. Therefore, sample of patients generated for analysis are from two patient groups: disease (defined by disease) and mimics (defined by mimics ). Patient groups are further classified into $\boldsymbol{P t}_{\text {type }}$ categories such that $k \in$ $\left(1,2, \ldots,\left|\boldsymbol{P} t_{\text {type }}\right|\right)$ based on patient presenting conditions. The classification is done based on the results of clinical tests for disease diagnosis in A\&E. The patient type category of $i^{\text {th }}$ patient is mathematically represented as:

$$
\begin{gathered}
\boldsymbol{P}_{\text {type }}(i)=k: k \in\left(1,2, \ldots,\left|\boldsymbol{P t}_{\text {type }}\right|\right) \quad \forall i \in\left(1,2, \ldots, N_{p t s}\right) \\
\boldsymbol{P t}_{\text {type }}=\text { disease } \cup \text { mimics } \\
\text { disease } \cap \text { mimics }=\varnothing
\end{gathered}
$$

\subsubsection{Modelling push and pull factors to simulate patient flow on care pathway}

In this step, push and pull factors between speciality units that are crucial for analysing patient diversions are modelled. Following sub-steps are used for developing a generic pathway model for pathway variation analysis:

\section{Push factors:}

A. Decision makers: In this sub-step, the effect of decision makers involved in making critical decisions (CD) within care pathway are mathematically modelled for analysis. The information about identifying critical decisions $(\boldsymbol{C D})$ is provided in Section 2.3. The effect of decision making factor is classified into regular work hours and after-work hours. During after-work hours, hospital has reduced specialist staff for diagnosis which reduces the quality of critical decisions (represented in $\boldsymbol{C D}$ ), thus resulting in more patients getting diverted to other speciality wards. As a result, the performance of clinical decision making in care pathway is lowered during after working hours. Patient arrival time $\boldsymbol{P t}_{\text {arrival }}(i) \forall i \in$ 
$\left(1,2, \ldots, N_{p t s}\right)$ is classified into patient arriving in ED in regular working hours and after work hours. A decision variable decision arrival $(i) \forall i \in\left(1,2, \ldots, N_{p t s}\right)$ is defined to classify patients arriving in regular work hours and after work hours. Mathematically,

$$
\begin{aligned}
& \text { decision }_{\text {arrival }}(i)=\left\{\begin{array}{cc}
1 & \text { if } W_{\text {start }} \leq \boldsymbol{P t}_{\text {arrival }}(i) \leq W_{\text {end }} \\
0 & \text { Otherwise }
\end{array}\right. \\
& \forall i \in\left(1,2, \ldots, N_{p t s}\right)(4)
\end{aligned}
$$

where, $W_{\text {start }}$ and $W_{\text {end }}$ are start and end time of the regular working hour of a shift. The outcome of decision $_{\text {arrival }}(i)$ of $i^{\text {th }}$ patient classifies patient arrival in regular and after working hours.

B. Clinical Decision Making Process: Research studies related to clinical decision making in case of care pathway generally involves diagnostic test performance assessment based on metrics derived from the confusion matrix. Clinical tests are assumed to be a binary classification model which classifies each patient into two classes: a true class (a disease is present) and a false class (disease in not present). More details on application of confusion matrices for the proposed study can be found in the supplementary material.

In case of complex integrated care pathways, several tests are performed before making final decision about putting patients on the care pathway. Therefore, confusion matrix is to be constructed for overall decision making. The patients data comprising of ward level journey information (see supplementary material for detailed list of data variables used) is used for constructing confusion matrix. Based on these confusion matrices, probability of sending patients correctly to their ward is evaluated (see supplementary material).

The performance of clinical decision making is determined with the help of probabilities of sending $k^{\text {th }}$ type patient $\left(k \in\left(1,2, \ldots,\left|\boldsymbol{P} \boldsymbol{t}_{\text {type }}\right|\right)\right)$ to a ward of care pathway during regular work hours $\left(\boldsymbol{P}^{\text {Reg }}\right)$ and after work hours $\left(\boldsymbol{P}^{\text {Aft }}\right)$. Mathematically,

$$
\begin{aligned}
\boldsymbol{P}^{\text {Reg }} & =\left[\alpha_{1}, \alpha_{2}, \alpha_{3}, \ldots \alpha_{k} \ldots, \alpha_{\left|P t_{\text {type }}\right|}\right] \\
\boldsymbol{P}^{\text {Aft }} & =\left[\beta_{1}, \beta_{2}, \beta_{3}, \ldots \beta_{k} \ldots, \beta_{\mid P t_{\text {type }}}\right]
\end{aligned}
$$

where, $\alpha_{k}$ and $\beta_{k}$ are estimated based on the confusion matrix detailed in supplementary material.

In simulation model, patients are sent to speciality ward based on arrival times, presenting conditions, and decision making probabilities identified based on confusion matrices. More mathematical formulation about these decisions are provided in the supplementary material. 
A decision variable decision $_{\text {prob }}(i)$ is defined based on $i^{\text {th }}$ patient arrival (i.e., decision $\left._{\text {arrival }}(i)\right), \boldsymbol{P t}_{\text {type }}(i)$, and $\boldsymbol{P}^{\boldsymbol{R e g}} \& \boldsymbol{P}^{A f t}$. $\boldsymbol{d e c i s i o n}_{\text {prob }}(i)$ selects probability from $\boldsymbol{P}^{\boldsymbol{R e g}} \& \boldsymbol{P}^{\boldsymbol{A f t}}$ based on patient type $\boldsymbol{P t}_{\text {type }}(i)$ and arrival type defined by $\boldsymbol{d e c i s i o n}_{\text {arrival }}(i)$. Mathematically,

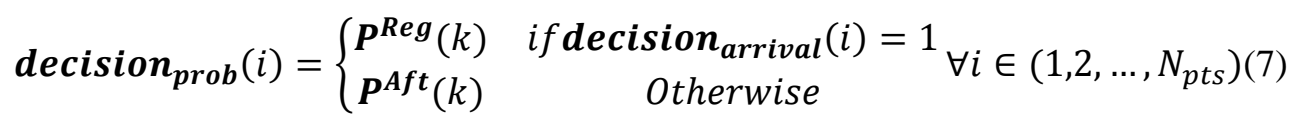

where, $\boldsymbol{P}^{\boldsymbol{R e g}}(k)=\alpha_{k}, \boldsymbol{P}^{\boldsymbol{A f t}}(k)=\beta_{k}$, and $\boldsymbol{P} \boldsymbol{t}_{\text {type }}(i)=k$.

Now, the decision to send a patient to ward included in care pathway of hospital based on the decision variable $\boldsymbol{d e c i s i o n}_{\text {prob }}(i)$ is mathematically represented as:

$$
\text { decision }_{\text {SendProb }}(i)=\left\{\begin{array}{lc}
1 & \text { if } R \leq \text { decision }_{\text {prob }}(i) \\
0 & \text { if } R>\text { decision }_{\text {prob }}(i)
\end{array} \forall i \in\left(1,2, \ldots, N_{p t s}\right)\right.
$$

where, $R$ is a random number $\in[0,1]$. decision SendProb $(i)=1$ simulates that $i^{\text {th }}$ patient is sent to wards included in care pathway based on the disease diagnosis and decision $_{\text {SendProb }}(i)=0$ means $i^{\text {th }}$ patient is sent to ward which is not included in the care pathway of hospital. Following decision variable defines each incoming patients into disease or mimics.

$$
\text { decision }_{\boldsymbol{P t s}}(i)=\left\{\begin{array}{ll}
1 & \forall \boldsymbol{P}_{\text {type }}(i) \in \text { disease } \\
0 & \forall \boldsymbol{P}_{\text {type }}(i) \in \text { mimics }
\end{array} \forall i \in\left(1,2, \ldots, N_{p t s}\right)\right.
$$

Abovementioned decision variables are used in next sub-step for estimating overall variations from care pathway in hospital.

C. A\&E 4-hour Operational KPI: As ED (Emergency Department or A\&E) is common entry point for most of the care pathway, hence operations in ED are modelled in this sub-step. When a patient arrives at ED, clinical interventions are carried out such as: triage, senior intervention following triage (SIFT), ED specialist registrar (SpR) assessment, ED consultant assessment. These interventions for $i^{\text {th }}$ patient coming to ED is collectively defined by $\boldsymbol{P} \boldsymbol{t}_{\boldsymbol{T} W}(i)$. The main components of $P t_{T W}(i)$ of $i^{\text {th }}$ patient are defined as: Triage time $\left(\boldsymbol{P} \boldsymbol{t}_{\text {Triage }}(i)\right)$, SIFT process $\left(\boldsymbol{P} \boldsymbol{t}_{\boldsymbol{S I F T}}(i)\right)$, ED SpR assessment time $\left(\boldsymbol{P t}_{\boldsymbol{E D S p R}}(i)\right)$, ED consultant assessment time ( $\left.\boldsymbol{P} \boldsymbol{t}_{\text {Consltant }}(i)\right)$, where $i \in\left(1,2, \ldots, N_{p t s}\right)$. These subinterventions time duration of $P t_{T W}(i)$ are mathematically defined as:

$$
\begin{aligned}
& \boldsymbol{P} \boldsymbol{t}_{\boldsymbol{T W}}(i)=\boldsymbol{P} \boldsymbol{t}_{\text {Triage }}(i)+\boldsymbol{P} \boldsymbol{t}_{\text {SIFT }}(i)+\boldsymbol{P t}_{\text {EDSpR }}(i)+\boldsymbol{P} \boldsymbol{t}_{\text {Consltant }}(i) \\
& \boldsymbol{P t}_{\text {Triage }}(i) \sim D\left(\mu_{\text {triage }}, \sigma_{\text {triage }}\right) \\
& \boldsymbol{P t}_{\text {SIFT }}(i) \sim D\left(\mu_{\text {SIFT }}, \sigma_{\text {SIFT }}\right) \\
& \boldsymbol{P t}_{\text {EDSpR }}(i) \sim D\left(\mu_{E D S p R}, \sigma_{E D S p R}\right)
\end{aligned}
$$

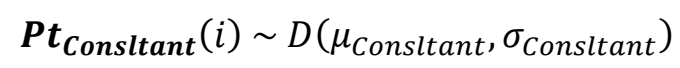


where, $D(\mu, \sigma)$ is a distribution having $\mu$ as its mean and $\sigma$ as standard deviation and $D\left(\mu_{\text {triage }}, \sigma_{\text {triage }}\right), D\left(\mu_{\text {SIFT }}, \sigma_{\text {SIFT }}\right), D\left(\mu_{E D S p R}, \sigma_{E D S P R}\right), D\left(\mu_{\text {Consltant }}, \sigma_{\text {Consltant }}\right)$ are assumed to be distributions of Triage time, SIFT time, EDSpR assessment time, and ED consultant assessment time.P $\boldsymbol{t}_{\boldsymbol{T W}}(i)$ can be modified for particular care pathway by adding or subtracting sub-intervention times in Eqn. (16) for patient sub-groups $\left(\boldsymbol{P}_{\text {type }}(i)\right)$ classified as priority or non-priority in care pathway.

The 4-hour operational KPI in ED is common across NHS trusts in UK due to Department of Health (DoH), UK guidelines for acute care in ED. It suggests that no patients in A\&E should wait more than 4 hours in A\&E from arrival to discharge or hospital admission. This leads to the push of patients to speciality units after arrival in ED. The time interventions window $\left(\boldsymbol{P} \boldsymbol{t}_{\boldsymbol{T} W}(i), i \in\left(1,2, \ldots, N_{p t s}\right)\right)$, is related to 4-hour operational KPI as it effects patient diversions from care pathway. to incorrect speciality units. Therefore, a binary decision variable $\operatorname{decision}_{\boldsymbol{E D K P I}}(i) \forall i \in\left(1,2, \ldots, N_{p t s}\right)$ is defined to consider patients breaching 4hour KPI of ED. Mathematically,

$$
\text { decision }_{\text {EDKPI }}(i)=\left\{\begin{array}{ll}
0 & \text { if } \boldsymbol{P t}_{T W}(i)>4 \\
1 & \text { if } \boldsymbol{P t}_{T W}(i) \leq 4
\end{array} \forall i \in\left(1,2, \ldots, N_{p t s}\right)\right.
$$

Therefore, $i^{\text {th }}$ patient sent to speciality units or wards correctly or incorrectly is determined by $\boldsymbol{P} \boldsymbol{t}_{\text {Sentward }}(i)$, which is mathematically represented by

$$
\text { Pt }_{\text {SentWard }}(i)=\operatorname{decision}_{\text {EDKPI }}(i) \times \text { decision }_{\text {SendProb }}(i)
$$

where, $\boldsymbol{P} \boldsymbol{t}_{\text {SentWard }}(i)=1$ represents that $i^{\text {th }}$ patient is sent to speciality unit correctly, and $\boldsymbol{P} \boldsymbol{t}_{\text {SentWard }}(i)=0$ represents that $i^{\text {th }}$ patient is sent to speciality unit incorrectly, following medical decision making.

\section{Pull factors:}

D. Ward Resources: The availability of resources (such as staff, beds) in speciality units within care pathway helps to admit patients from preceding speciality units. However, lower availability of resources can cause patients not being admitted to speciality unit and results in patient diversions. Therefore, capacity constraint of speciality units in care pathways can be a factor affecting patient diversions. Resource utilization is modelled as a key performance indicator (KPI) of speciality wards. In this paper, we have considered number of beds as the speciality unit's capacity. However, other resource parameters such as staff/equipment can be also used for determining speciality unit's capacity.

Beds in Speciality Wards: Let us consider bed capacity of a speciality unit be $B_{S U}$ and average patient length of stay in speciality unit be $\operatorname{LoS}_{S U}$ (in days). Then bed utilization in a speciality unit can be approximated as: 


$$
U_{S U}^{B e d}=\frac{L_{S} S_{S U} \times \sum_{i=1}^{N_{p t s}} \boldsymbol{P} t_{\text {SentWard }}(i)}{B_{S U} \times T} \times 100
$$

where, $\sum_{i=1}^{N_{p t s}} \boldsymbol{P} \boldsymbol{t}_{\text {SentWard }}(i)$ represents number of patients sent correctly to speciality unit in time duration $T$ (in days).

E. Key Performance Indicator (KPI) of speciality units: There can be KPI of speciality units, which helps speciality units to receive financial incentives by providing care to patients in a pathway. This results in pull to admit patients from preceding units to improve KPI of the speciality unit.

The push factors such as decision makers, clinical decision making process, and 4-hour operational KPI of ED ensures that patients are referred (push) to speciality units, however, capacity constraints of a speciality unit can restrict patient admissions to the speciality unit. As a result, patients are sent to other speciality units or medical wards incorrectly after ED. Therefore, patient diversions from care pathways can be mathematically represented as:

$$
\begin{aligned}
\operatorname{Max}\left(\text { Sent }_{S U}=\right. & \left.\sum_{i=1}^{N_{p t s}} \boldsymbol{P t}_{\text {SentWard }}(i) \times \text { decision }_{\text {Pts }}(i)\right) \\
& \text { Subject to: } U_{S W}^{B e d} \leq 80 \%
\end{aligned}
$$

$\mathrm{Eq}^{\mathrm{n}}$. (24) represents objective function which maximises the number of patients being sent to speciality units correctly. Constraint represented in Eqn. (25) represents that the utilization of beds in a specialty unit must be less than $80 \%$. This formulation based on push and pull factors will help to reduce patient diversions and simultaneously monitor the specialty unit's capacity to accommodate increased number of patients referred from ED.

\subsubsection{Analysis of Pathway Variations Simulation Model}

In this step, simulation analysis is performed to simulate patient flow in a care pathway. Monte Carlo simulation is used to simulate patient flow. The main objective of this step is to determine various improvement options to reduce patient diversions from care pathway.

A. Estimating inputs and running the simulation model:

The inputs to the simulation model such as patient arrival distributions, process step (triage, SIFT, SpR time, consultant time) duration distributions in ED and probabilities represented in $\boldsymbol{P}^{\boldsymbol{R e g}} \& \boldsymbol{P}^{\boldsymbol{A f t}}$. Hospital IT systems largely stores information such as timestamp data for process steps and patient's ward level journey data which is used to identify closely fitting distributions. These inputs are then used for simulation.

B. Analysis of simulation model for set-based improvements:

The simulation models developed for healthcare services generally focuses on suggesting single parameter change (or individual change) type solutions for service improvements (such as changing number of beds, staff, or removing certain tasks which causes delays) [29-31]. 
The single parameter change solution means suggesting changing one parameter in the simulation model to generate 'what-if' scenarios. However, single parameter change solution does not consider the interactions among other parameters as a result. Hence, single parameter change solutions do not work for inter-related services such as integrated care pathways in hospital. Hence, the simulation model for integrated care pathway is analysed with the help of set-based solutions.

\subsection{Problem identification in care pathway}

The IT systems data (the variable names are presented in supplementary material) is used to monitor the performance of care pathways based on crucial key performance indicator (KPI). When the performance target of care pathway is not met, then this step is used to identify problems in care pathway. The details about these problems are:

\subsubsection{Low throughput in care pathway}

One of the problems in care pathway within hospital is low throughput of patients treated within care pathways. The lower throughput of care pathway within hospital is mainly due to bottlenecks in one or more service delivery specialty units along the care pathway. Solving care pathway throughput issue can only improve patient flow for patients who are correctly placed on care pathway. In this paper, we focus on patient diversions from care pathway, therefore, more information about throughput issue or bottleneck identification is not discussed.

\subsubsection{Patient diversions from care pathway}

Patient diversion from care pathway is identified to be a major problem for integrated care pathway of hospital. In this sub-step, a systematic procedure for identifying decisions that causes patient diversions from care pathway is discussed based on RAD model and historical patient's data.

Following is the two-step process to identify pathway variations.

\section{A. Identifying candidate set of decisions in RAD leading to pathway variations:}

The decisions involved within care pathway are graphically illustrated in RAD as a notation called case refinement. These candidate case refinements are mathematically represented as:

$$
\boldsymbol{C R}=\left[C R_{1}, C R_{2}, \ldots, C R_{|C R|}\right]
$$

Figure 3 highlights decisions, within a simple process represented in RAD, that results in patient being sent to ward A, B, or C, or classified as other patients (patients not eligible for treatment/care on specialised care pathway). The definition of RAD symbols is provided in Table A.1 in Appendix A. Based on the RAD represented in Fig. 3, a conceptual decision making representation is developed (see Fig. 4). 
Figure 4 represents the case refinements $(\boldsymbol{C R})$ present in the RAD of care pathways. Circles in Fig 4 represent the highlighted case refinements.

B. Identifying critical decisions from $\boldsymbol{C R}$ : The candidate set of decisions $(\boldsymbol{C R})$ are analysed in this sub-step to identify critical decision(s) that is (are) significantly affecting patient diversions from pathway. The candidate decisions are mapped to ward-level locations to provide mapping between decisions in $\boldsymbol{C R}$ and their resulting impact on patient ward level flow. This is analysed with the help of patient pathway data from hospital IT system (see supplementary material for more information). Mathematically, mapping is defined based on matrix $\mathbf{L}=\left[l_{a, b}\right]_{a=1,2, \ldots, w ; b=1,2, \ldots, w}$.

$$
l_{a, b}=\left\{\begin{array}{cc}
c & \text { if } C R_{c} \in \boldsymbol{C R}: C R_{c}=\operatorname{decision}(a \rightarrow b) \\
0 & \text { if decision }(a \rightarrow b) \notin \boldsymbol{C R} \\
0 & \text { if } a=b
\end{array}\right.
$$

where, $c \in[1,|\boldsymbol{C R}|], a, b$ represents ward-level locations of patient and $\operatorname{decision}(a \rightarrow b)$ represents decisions in $\boldsymbol{C R}$ leading to patient being sent to ward $b$ from ward $a$. $w$ is the total number of wards patients can go to, which can be significantly reduced by grouping incorrect wards as one type of ward.

Once, matrix $\mathbf{L}$ is constructed, the impact of decisions in $\boldsymbol{C} \boldsymbol{R}$ is quantified based on the EPR containing patient ward level movement data. Mathematically, impact of $C R_{c}$ is assessed as:

$$
I_{C R_{c}}=\frac{\sum_{r=1}^{R} d_{c_{a}, c_{b}}^{r}}{\sum_{r=1}^{R} \sum_{a=1}^{W} \sum_{b=1}^{W} d_{a, b}^{r}}
$$

where, $I_{C R_{C}}$ is impact of $C R_{c}$ on patient pathway based on patient records $r \in[1, R]$. Total number of patient records from hospital EPR (for a given time period) is defined as $R$. Indices $c_{a}$ and $c_{b}$ defines the row and column of $\mathbf{L}$ which has attribute value $c(c \in[1,|\boldsymbol{C R}|])$. And, $d_{c_{a}, c_{b}}^{r}$ is defined as:

$$
d_{a, b}^{r}=\left\{\begin{array}{cc}
1 & \text { if pt }(a \rightarrow b) \text { in } r^{\text {th }} \text { patient record } \\
0 & \text { else }
\end{array}\right.
$$

where, $p t(a \rightarrow b)$ defines patient sent to ward $b$ from ward $a$.

The impact of each decision in $\boldsymbol{C} \boldsymbol{R}$ is assessed based on $I_{C R_{c}}$ defined in Eqn. 28. $C R_{C}$ The case refinements which significantly leads to patient diversions from the care pathway is selected as critical decision $(\boldsymbol{C D})$ based on threshold $T h$ (if $I_{C R_{c}} \geq T h$ then $C R_{C} \in \boldsymbol{C D}$ ). The critical decisions $(\boldsymbol{C D})$ are 
then used in subsections 2.1.2 clinical decision making process modelling, following clinical decision making modelling, pathway variations are analysed based on Section 2.2.

\section{Case Study}

The case study involves the stroke care pathways (SCP) for acute ischemic stroke (AIS) at a large UK hospital (with more than 1200 patient beds). Stroke represents a major health problem worldwide with an estimated stroke care costs NHS $£ 2.8$ billion a year in direct care costs [42]. Furthermore, over 300,000 people live with moderate to severe disabilities as a result of stroke in UK [43]. As a result, various clinical guidelines have been introduced to improve the stroke care services by developing stroke care pathways [18-20].

AIS patients generally arrive at Accident and Emergency (A\&E) of a hospital from outside with the help of paramedics, emergency medical services (EMS) or ambulance services. Medical team in A\&E then assesses patient based on initial clinical observations and diagnostic imaging. Once AIS case is identified in a patient, acute stroke team of Neurology assesses patients for hyper acute monitoring and treatment (e.g. thrombolysis). After hyper acute phase, patients are shifted to acute stroke wards for rehabilitation and physiotherapy treatments. Finally, the process ends when the patients are discharged from the hospital. As such, stroke care pathway within a hospital deals with multiple departments such as Accident \& Emergency (A\&E), Neurology, Radiology, and Lab Services. Several IT systems storing EPR including patient administration system (e.g. iPM), beds management system (e.g. Extra-Med), clinical reporting system (e.g. CRS) in A\&E, radiology information system (e.g. RIS) in Radiology and clinical system (e.g. Dendrite) in Neurology is used for storing stroke patients care related information.

Stroke care pathway in a hospital can be divided into: (i) hyper acute stroke phase, and, (ii) acute stroke phase. This case study deals with the hyper acute stroke phase to illustrate the application of the proposed approach for identifying and solving patient diversion problem. Figure 5 illustrates the hyper acute process which is divided into: patient arrival process, medical team assessment, neurological assessment, and hyper acute stroke ward process.

The proposed variations modelling methodology was applied to model, simulate and analyse the hyper acute phase of stroke care pathway of a hospital. The subsections below detail the application of the proposed methodology to stroke care pathway.

\subsection{System-level modelling of stroke care pathway}

In this section, the RAD model of hyper acute phase of stroke care pathway was developed based on interviews of staff involved in stroke care pathway in a hospital. Readers are referred to [17] for information on systematic methodology for RAD model development from staff interviews.

\subsubsection{Stroke care pathway modelling}

The hyper acute phase of stroke pathway is logically illustrated in Fig. 6. Below is the discussion about the RAD model developed for hyper acute phase of stroke care pathway. 
Figure 6 illustrates four sub-processes of hyper acute phase of stroke care pathway. Patient arrival process is divided into: (i) FAST (Facial weakness, Arm weakness, Speech problems, Time to call Emergency number) positive patient arrival process, and, (ii) Non-FAST positive patient arrival and medical team assessment process. FAST is a type of clinical assessment performed by Paramedics or Triage Nurses for identifying stroke patients and if positive, pre-alerting acute stroke team (neurology registrar, stroke nurse) while transporting patients to local A\&E. If a patient is FAST positive, paramedic staff pre-alerts acute stroke team and then patients are rapidly assessed by stroke team upon arrival at A\&E. However, if a patient is non-FAST positive, then patients undergo medical team assessment upon arrival at $A \& E$ and then acute stroke team is notified if initial clinical assessment confirms stroke. Both FAST positive and non-FAST positive patients then undergo neurological assessment for stroke confirmation followed by hyper acute stroke ward process where various treatments are provided to respective stroke patients. The detailed RAD for each of the sub-process steps and its details are provided in Appendix A (Figs. A.1 - A.4).

The RAD models of stroke care pathways developed were used in next subsection to identify critical decisions leading to patient variations from pathways.

\subsection{Problem identification in stroke care pathway}

In this step, the performance of stroke care pathway is monitored based on the patient data available in hospital. The crucial key performance indicator of the stroke care pathway which stroke services are struggling to achieve is $80 / 90 \mathrm{KPI}$. The $80 / 90 \mathrm{KPI}$ is defined as more than $80 \%$ of total stroke patients coming to the hospital must spend more than $90 \%$ of their hospital stay in stroke speciality units. A number of randomized clinical trials are consistently yielding findings wherein acute stroke patients who spend most of their time at a hospital in a stroke ward/stroke unit experience better recovery outcomes [45]. Based on these findings, UK DoH [19, 20] stipulates that $80 \%$ of stroke patients must spend 90\% of their stay at a hospital in a stroke ward / stroke unit (80/90 KPI). Therefore, hospitals attempting to adhere to this performance measure must transport acute stroke patients into a stroke ward immediately upon confirmation of the diagnosis in A\&E. Hence, in this paper we will use 80/90 KPI to evaluate the performance of overall stroke care pathway. The performance of stroke care pathway in hospital in previous months was lower than the 80/90 KPI. More information on it has been provided later in this section.

The main reason for the stroke care pathway in hospital not being able to achieve targeted performance was largely due the patient diversions to other non-stroke speciality units, thus significantly reducing stroke patient's length of stay in stroke units. Therefore, in this section, decisions leading to significant patient diversion from the stroke care pathway are identified.

The decision making steps that leads to variations from the pathways is identified based on following sub-steps:

\subsubsection{Identifying candidate decisions from system model of stroke pathway}


As discussed in Section 2.3.2, candidate case refinements or decision making steps from RAD model of stroke care pathway are identified which was leading to pathway variation. These decisions are Is patient suspected of suffering stroke?, Patient suffered stroke?, Patient breaching $4 \mathrm{Hr}$ AE KPI? in Fig. A.2, and, Is patient suffering from Stroke? in Fig. A.4. These candidate set of decisions (represented in Fig. 7) was further analysed in next step with the help of patient records.

\subsubsection{Identifying critical decision from $\boldsymbol{C R}$}

The EPR data for stroke patients was analysed to identify the impact of the decisions on pathway variations. Stroke patient referrals from A\&E and other sources to stroke wards (speciality unit involved in providing stroke care within stroke care pathway), clinical decisions unit (CDU), and other wards such as cardiology wards (CRD), medical wards (MED), surgery wards (SRG), other wards (RGB, W40) are computed and are represented in Fig. 8. CDU and other wards are not involved in providing specialist care to stroke patients and thus stroke patients referred to these units were considered as patient diversion from stroke care pathway. Figure 8 illustrates the pathway variations from stroke pathway in hyper acute and acute phase based on 5 months of hospital data. The red coloured boxes indicate the non-stroke wards and green colour box represent the stroke wards. The red arrows represent stroke patient diversions and blue arrows indicate correct referral of stroke patients. Therefore, red arrows represent patient diversions in Fig. 8. Significant number of stroke patients is diverted to CDU after A\&E in Fig. 8 (97 from $A \& E$ and 2 from other sources stroke patients sent to CDU). These patient diversions led to less number of stroke patients spending most of their hospital stay in stroke speciality units.

Decisions Patient breaching 4Hr AE KPI? and Is patient suffering from Stroke? are usually taking place in A\&E and leads to patients being diverted to CDU (see Fig. 7). Hospital wards are grouped into A\&E, Stroke Wards (W41, W42, W43), CDU, and Other Wards (W40, SRG, CRD, MED, RGB) (i.e.,w $=4)$. Therefore, the impact $\left(I_{C R_{c}}\right)$ for both of these decisions is computed as:

$$
I_{C R_{c}}=\frac{\sum_{r=1}^{298} d_{1,3}^{r}}{\sum_{r=1}^{298} \sum_{a=1}^{4} \sum_{b=1}^{4} d_{a, b}^{r}}=\frac{97}{97+4+27+2+131+2+8+1} \approx 0.356
$$

where, $d_{1,3}^{r}$ represents decisions leading patient being sent to CDU from A\&E.

Decisions Is patient suffering from Stroke? and Patient breaching 4Hr AE KPI? are eligible as critical decision (CD) leading to significant patient diversions as these result in major patient diversions. Decisions Is patient suffering from Stroke? and Patient breaching $4 \mathrm{Hr}$ AE KPI? were considered as one because both of them are leading to same type of patient variation (i.e., from $\mathrm{A} \& \mathrm{E}$ to $\mathrm{CDU})$ and there is lack of data supporting individual decision analysis. These decisions were analysed in next sub-step based on the interview of Neurology Registrar and Medical Team 
(in $\mathrm{A} \& \mathrm{E}$ ) about decision making process for identification of stroke in $\mathrm{A} \& \mathrm{E}$ and their referral to stroke wards, CDU, and other wards.

\subsection{Decision making process modelling}

In this sub-section, neuro $S p R$ and ED medical team were interviewed about the decision making process involving other roles, clinical information, clinical tests, and patient medical history for identifying stroke. Figure 9 illustrates the RAD model for decision making process adopted by neurology registrar and medical team for identifying stroke. In Fig, 9, it can be seen that the neurology $S p R$ or medical team doctor will first assess patients based on triage notes or FAST (face weakness, arm weakness, speech, time) test notes. Initial assessment including vital signs and medical history is assessed for suspected stroke patients.

For suspected stroke patients, symptom progression, time onset, focal neurological deficits, and vascular risk factors are evaluated to identify whether patient presenting condition suggest stroke. If these assessments suggest stroke, then differential diagnosis such as ranking based on NIHSS (national institute of health stroke scale), ECG and Blood test, and CT scan are performed to confirm stroke and assess patient treatment options. Based on these assessments, patients are sent to stroke wards or CDU or other wards. RAD model developed in this step was used to analyse the pathway variations analysis in next step.

\subsection{Pathway variation analysis (PVA) for stroke care pathway}

The stroke care pathway was analysed based on the pathway variation analysis (PVA) methodology discussed in Section 2.2. Details of steps involved in PVA for stroke care pathway are described as follows:

\subsubsection{Generate sample of patients for analysis}

The sample of patients was generated based on following parameters:

A. Sample size: The time period time period $T=30$ days is used and number of patients is identified to be $N_{p t s} \sim N(105,9)$.

B. Patient arrival time: The distribution for patient arrival time to A\&E (Pt $\left.\boldsymbol{t}_{\text {arrival }}\right)$ (see Fig. 10) is identified to be:

$$
\boldsymbol{P t}_{\text {arrival }} \sim N(13.8,5.28)
$$

C. Patient presenting conditions: $\boldsymbol{P t _ { \text { type } }}$ is identified with the help of RAD model of the critical decision making process obtained in Section 3.3. Each patient type is based on patient clinical test results that are performed by neurology registrar and medical team to identify stroke. Five sub-groups of patients (stroke and stroke mimics) are identified based on major clinical assessment tests such as FAST, focal neurological deficit (FND), symptom onset type, and TIA history. For e.g., $1^{\text {st }}$ type of patients have FAST test as positive, $2^{\text {nd }}$ type of patient have FAST test as negative and FND test as positive, $3^{\text {rd }}$ type of patient have FAST, FND test as negative, symptom onset type, TIA history as positive, $4^{\text {th }}$ type of patients have FAST, FND 
test as negative, and symptom onset type, TIA history as negative, and, $5^{\text {th }}$ type of patients have all the stroke tests as negative. Therefore, stroke $=\{1,2,3,4\} \&$ mimics $=\{5\}$ represents stroke patients and stroke mimics patients category. Therefore,

$$
\boldsymbol{P t}_{\text {type }}=\{1,2,3,4,5\}: \text { stroke }=\{1,2,3,4\}, \text { mimics }=\{5\}(26)
$$

Based on abovementioned parameters, a population of patients with stroke and stroke mimics was created.

\subsubsection{Modelling push and pull factors to simulate of patient flow on stroke care pathway}

As defined in Section 2.2.2, the push and pull factors among speciality units involved in stroke care pathway leads to patient diversions. These factors were modelled and were estimated based on patient data. These factors are:

\section{Push Factors:}

A. Decision makers in Regular work hours and after work hours: The quality of decisions is affected during the regular work hours (8AM - 4PM) and after work hours. This is due to the presence of neurologist in regular work hours and only medical team in A\&E together with neurologist 'on call' service takes over during after work hours.

B. Evaluation of decision making process for stroke: The probabilities for sending stroke or stroke mimics patients to stroke wards during regular and after hours are identified from 3 months of hospital data (see Section 2.2.2.B):

$$
\begin{aligned}
P^{R e g} & =[0.95,0.55,0.80,0.70,0.05] \\
P^{A f t} & =[0.95,0.44,0.70,0.50,0.10]
\end{aligned}
$$

C. A\&E 4 Hour Operational KPI: The time distributions for clinical interventions in A\&E are identified for $\boldsymbol{P} \boldsymbol{t}_{\text {Triage }}, \boldsymbol{P} \boldsymbol{t}_{\text {SIFT }}, \boldsymbol{P} \boldsymbol{t}_{\text {EDSPR }}, \boldsymbol{P} \boldsymbol{t}_{\text {Consltant }}$ in hours. These distributions are identified and represented as:

$$
\begin{gathered}
\boldsymbol{P t}_{\text {Triage }} \sim \operatorname{Unif}(0.4,0.6), \boldsymbol{P t}_{\text {SIFT }} \sim \operatorname{Unif}(0.7,1.0) \\
\boldsymbol{P \boldsymbol { t } _ { \text { EDSPR} }} \sim \operatorname{Unif}(1.0,1.5), \boldsymbol{P t _ { \text { Consltant } }} \sim \operatorname{Unif}(1.2,2.2)
\end{gathered}
$$

Based on the distributions of $\boldsymbol{P t} t_{\text {Triage }}, \boldsymbol{P t} t_{S I F T}, \boldsymbol{P t} t_{E D S p R}, \boldsymbol{P t} t_{\text {Consltant }}$, the $\boldsymbol{P t _ { T W }}(i)$ for each of the $i^{\text {th }}$ patient was generated. Furthermore, based on the hospital current policy about stroke care, $\boldsymbol{P t}_{\boldsymbol{T} \boldsymbol{W}}(i)$ is defined as:

$$
\boldsymbol{P t}_{\boldsymbol{T W}}(i)=\left\{\begin{array}{cc}
\boldsymbol{P t}_{\text {Triage }}(i) & \forall \boldsymbol{P}_{\text {type }}(i)=1 \\
\boldsymbol{P t}_{\text {Triage }}(i)+\boldsymbol{P t}_{\text {SIFT }}(i)+\boldsymbol{P t}_{\text {EDSp } \boldsymbol{R}}(i)+\boldsymbol{P t}_{\text {Consltant }}(i) & \text { Otherwise }
\end{array}\right.
$$

$i^{\text {th }}$ patient such that $P_{\text {type }}(i)=1$ represents patient category defined as FAST positive patients, which are referred directly to Acute Stroke Team after triage in ED and thus, $\boldsymbol{P} \boldsymbol{t}_{\boldsymbol{S I F T}}(i), \boldsymbol{P} \boldsymbol{t}_{\boldsymbol{E D S p R}}(i), \boldsymbol{P} \boldsymbol{t}_{\text {Consltant }}(i)$ times are not included in $\boldsymbol{P} \boldsymbol{t}_{\boldsymbol{T W}}(i)$ in Eqn. (11).

As discussed in section 2.2.2.C, $\boldsymbol{P} \boldsymbol{t}_{\boldsymbol{T W}}(i)$ is used as a parameter to assess the 4-hour A\&E operational KPI.

\section{Pull Factors:}


D. Bed utilization KPI of hype acute stroke Ward: The bed utilization KPI is computed based on hyper acute bed capacity in hyper acute stroke ward. Currently, hyper acute stroke ward has 4 beds (i.e., $B_{S U}=4$ ) and $L o S_{S U} \approx 3.25$ (estimated using hospital patient records data). Therefore, $U_{S U}^{B e d}$ is estimated as:

$$
U_{S U}^{B e d}=\frac{3.25 \times \sum_{i=1}^{N_{p t s}} \boldsymbol{P t}_{\text {SentWard }}(i)}{4 \times T} \times 100
$$

E. Stroke Unit KPI: The 80/90 KPI of stroke unit of a stroke care pathway is identified as a crucial factor affecting stroke patients being sent to stroke unit. This KPI is evaluated based on Sent $_{S U}$ and Sent ${ }_{C D U}$ representing patients sent to stroke units and non-stroke units such as CDU. These parameters are used to identify percentage of patients spending more than more than $90 \%$ of their hospital stay in stroke wards $(O B J)$. Mathematically,

$$
O B J=\frac{f_{1} \times \operatorname{Sent}_{S U}+f_{2} \times \operatorname{Sent}_{C D U}}{\operatorname{Sent}_{S U}+\operatorname{Sent}_{C D U}} \times 100
$$

Parameters $f_{1}$ and $f_{2}$ are evaluated based on the EPR database storing the stroke patient information.

\subsubsection{Analysis of stroke care pathway variations simulation model}

As discussed in section 2.2.3, Monte Carlo simulation technique was used for simulation analysis.

Following sub-steps further discusses the simulation analysis:

A. Running the simulation model for stroke care pathway:

For simulation modelling, a group of patients $\left(N_{p t s}\right)$ was created based on the distributions of each patient types $\left(\boldsymbol{P} \boldsymbol{t}_{\text {type }}\right)$. Then, $\boldsymbol{P} \boldsymbol{t}_{\boldsymbol{T} W}(i)$ for $i^{\text {th }}$ patient is estimated based on $\boldsymbol{P}_{\text {type }}(i)$. The decision which ward $i^{\text {th }}$ patient is sent to is calculated. Finally, number of stroke patients sent to stroke wards and CDU is computed. A simulation model is developed in MATLAB (2010) software, which follows the abovementioned procedure for large number of iterations. The number of stroke patients spending more than $90 \%$ of their stay in stroke wards is evaluated (as in Eq. 13).

The simulation model was then validated by taking additional three month data and comparing various key output parameters of the model with the raw data. The 80/90 KPI from the simulation model for additional three months was 74.6 (77.62 - 71.59 at 95\% Confidence Interval). The 80/90 KPI from the 3 months real data was 75.02, 73.68, and 71.31. Similarly, number of patients going to stroke wards, clinical decisions unit, and other wards was 36 (39 -33 at 95\% CI) , 19 (21 - 16 at 95\% CI), and 7 (10-5 at 95\% CI). These numbers were compared with the real data: average number of patients sent to stroke wards, clinical decisions unit, and other wards was 34,18 , and 7 . It this evident from this validation that the model is consistent with the newer datasets as well. The simulation model developed 
was then used in next step for suggesting set-based solutions to reduce patient diversion from stroke care pathway.

B. Analysis of stroke care pathway simulation model for set-based solutions:

After running the simulation model of pathway variations of current state, the current percentage of stroke patients spending more than $90 \%$ of their hospital stay in stroke wards is 73\% (Approx.). After running the simulation model for $T=30$ days, the utilization of stroke beds was estimated to be $U_{S U}^{B e d} \approx 81 \%$.

Improvement options are analysed and their potential realization in hospital scenario was determined. To suggest changes, average percentages of patient types are illustrated in Table 1. Table 1 shows the impact of patient types on overall number of stroke patients coming to hospital for stroke care. Patient type 1 to 4 is stroke patients and $5^{\text {th }}$ category was for stroke mimics patients (non-stroke patients having stroke like conditions).

From Table 1, patients with negative FAST test and positive FND test (i.e. $k=2, k \in$ $\boldsymbol{P} \boldsymbol{t}_{\text {type }}$ ) was $32.2 \%$ which is largest in stroke patients set ( $k \in$ stroke). Furthermore, probabilities of sending patients to stroke wards for $P_{\text {type }}(i)=2$ was $\boldsymbol{P}^{\boldsymbol{R e g}}=0.55$ and $\boldsymbol{P}^{\boldsymbol{A f t}}=0.44$ in regular and after hours. Therefore, improving the decision making for patients of $2^{\text {nd }}$ type, i.e., $P_{\text {type }}(i)=2$, can significantly improve number of stroke patients correctly sent to stroke wards, thereby improving $O B J$. However, increasing the number of stroke patients sent to stroke wards following their diagnosis in A\&E, will increase the number of patient coming to stroke wards for stroke care. Therefore, the capacity of stroke wards (i.e., number of beds) was increased simultaneously with the improvement of clinical decision making for $P_{\text {type }}(i)=2$. Hence, following improvement options are suggested.

Option A: In this option, patient of type 2, i.e., $P_{\text {type }}(i)=2$, are prioritized after triage in A\&E to reduce overall clinical assessment time $\left(\boldsymbol{P t} t_{T W}(i)\right)$ and training of A\&E staff about identifying stroke once patient is identified as FND positive. In simulation model, these changes will be reflected as reduced clinical intervention time for patient of type 2 and higher probabilities for sending stroke patients to stroke wards. That is mathematically represented as:

$$
\boldsymbol{P} \boldsymbol{t}_{\boldsymbol{T W}}(i)=\left\{\begin{array}{cc}
\boldsymbol{P t}_{\text {Triage }}(i) & \forall \boldsymbol{P}_{\text {type }}(i)=1 \\
\boldsymbol{P} \boldsymbol{t}_{\text {Triage }}(i)+\boldsymbol{P t}_{\text {Consltant }}(i) & \forall \boldsymbol{P}_{\text {type }}(i)=2 \\
\boldsymbol{P t}_{\text {Triage }}(i)+\boldsymbol{P} \boldsymbol{t}_{\text {SIFT }}(i)+\boldsymbol{P t}_{\text {EDSpR }}(i)+\boldsymbol{P} \boldsymbol{t}_{\text {Consltant }}(i) & \text { Otherwise }
\end{array}\right.
$$

And probabilities of type 2 patients in regular and after work hours were represented as $\boldsymbol{P}^{\boldsymbol{R e g}}(2)=0.75$ and $\boldsymbol{P}^{\boldsymbol{A f t}}(2)=0.65$ this was due to the improved decision making due to A\&E staff training on stroke patient identification if FND is positive. This improvement suggestion will help to get more stroke patients into stroke wards, hence, 
bed capacity of the stroke ward was increased in the analysis. Increasing, $B_{S U}=5$ (i.e., adding one extra bed in stroke ward) resulted in $U_{S U}^{B e d} \approx 79 \%$.

Option B: In this option, patient of type 2, i.e., $P_{\text {type }}(i)=2$ are fast tracked in A\&E (as was done for FAST positive patients, i.e., $\left.P_{\text {type }}(i)=1\right)$. That is, having reduced $\boldsymbol{P t _ { T W }}(i)$ by removing SIFT time, ED SHO/SpR/Doctor time, Consultant time and considering them as a high priority patients by pre-alerting for stroke diagnosis. Mathematically, $\boldsymbol{P t _ { T W }}(i)$ can be represented as:

$$
\boldsymbol{P} \boldsymbol{t}_{\boldsymbol{T W}}(i)=\left\{\begin{array}{cc}
\boldsymbol{P t}_{\text {Triage }}(i) & \forall \boldsymbol{P}_{\text {type }}(i)=1,2 \\
\boldsymbol{P t}_{\text {Triage }}(i)+\boldsymbol{P} \boldsymbol{t}_{\text {SIFT }}(i)+\boldsymbol{P t}_{\text {EDSpR }}(i)+\boldsymbol{P t}_{\text {Consltant }}(i) & \text { Otherwise }
\end{array}\right.
$$

Since, patients of type 2 in this improvement option are referred directly to acute stroke team after triage (using pre-alerts), therefore, the probability of sending patients increased (same as type 1 patient) due to better decision making (due to Neuro-SpR and stroke nurse involvement in acute stroke team). Hence, probabilities of type 2 patients in regular and after work hours are represented as $\boldsymbol{P}^{\boldsymbol{R e g}}(2)=0.95$ and $\boldsymbol{P}^{\boldsymbol{A f t}}(2)=0.95$. Implementation of this option will result in more number of stroke patients coming to stroke wards for stroke care, therefore, bed capacity was increased based on increment in stroke patient referred to stroke ward. Increasing $B_{S U}=6$ (i.e., adding two extra beds in stroke ward) resulted in $U_{S U}^{B e d} \approx 80 \%$.

Figure 11 illustrates the results of the pathway variations simulation analysis for current, option A and option B. Fig. 11 (I) shows the number of patients that are correctly sent to stroke ward, and Fig. 11 (II) shows the number of stroke patients that are incorrectly sent off to non-stroke wards verses number of hyper-acute beds available/required for current (4 beds), option A ( 5 beds), and option B (6 beds). Further, Fig. 11 (III) illustrates number of stroke patients incorrectly sent to non-stroke wards for current, option A, and option B, and Fig. 11 (IV) illustrates the percentage of stroke patients spending more than $90 \%$ of their hospital stay in stroke wards $(O B J)$ verses number of beds required for option A and option B. It is evident from Fig. 11, that by improving decision making process (i.e., option A and B) and increase of hyper acute bed capacity (i.e., from 4 to 5 and 6), the 80/90 KPI can increase from $73 \%$ to $81 \%$ and $93 \%$ respectively.

Based on the results of simulation analysis, set-based solutions were suggested to the hospital to reduce patient diversions from stroke care pathway, which helped hospital's stroke care program to meet their contractual 80/90 KPI. Table 2 illustrates the performance of the hospital against the 80/90 KPI.

\section{Conclusions}

Variations from care pathways have been identified as a major challenge in the success of process improvement studies within hospital. Therefore, a PVA methodology is developed for modelling and 
simulating care pathway variations to highlight substandard care pathways in terms of efficiency/effectiveness. The PVA methodology identifies major patient variations from care pathway such as patient diversions and develops a model of healthcare operations together with the goodness of decision making processes involved. The mathematical model thus developed is used for simulation analysis by analysing hospital data. Based on simulation analysis, improvement scenarios were suggested which helps in reducing pathway variations.

The main features of Pathway variation analysis (PVA) method can be summarised as: (i) identifying sources of unwarranted variations from care pathway such as patient diversions from care pathway based on accurate and scalable service delivery system model, (ii) modelling crucial factors such as decision making process, decision makers, operational parameters, and inter-departmental performance measures leading to patient pathway variations, (iii) approach to include the information about patient characteristics related to disease presentations and clinical tests for identifying patient diversions and its integration with service delivery system model, (iv) development of pathway variation analysis (PVA) methodology for identifying, modelling \& simulating pathway variations and then for suggesting set-based solutions to reduce patient unwarranted variations from care pathway and (v) implementation of proposed methodology on stroke care pathways for unwarranted patient variations reduction in a large UK Hospital.

Traditionally, variety of simulation models in the field of hospital process improvements has been developed. Nevertheless, most of these existing models are discrete event simulation to identify bottlenecks, resource utilisation, and patient/staff scheduling and lacks consideration for ineffective decision making which can cause patient transfers to wrong wards. The major weakness of discrete event simulation alone can be due to the use of simplified process diagrams and imperfect decision making processes. These simplifications can lead to misleading model based estimations.

Proposed pathway variations analysis model is based on process model, decision model, and simulation. Proposed integrated model has better ability to represent various operational constraints that is required in complex modelling scenarios such as stroke care pathways.

This paper has developed the model for pathway variations analysis following good modelling practice described in [49]. The proposed model has been verified internally by extensive logic reviews with domain experts. All of these were consistent with expected modelling parameters and assumptions. Various modules of the model are validated against external datasets, which were not used as an input for model parameterization. The model output seems coherent and does not significantly diverge from external datasets.

The proposed model has got some limitations. The process modelling part of the methodology requires conducting interviews with process participants, which can be time consuming. The model only considers number of beds a measure for ward capacity, which can be extended to include number of staff/equipment as well. The proposed model deals with one key performance measure at a time for variations analysis. Therefore, the model can be extended in case of multiple performance measures. 
The proposed individual level model requires lot of data for modelling various aspects such as push and pull factors. The model developed in this paper needs to be carefully verified and validated, which is time consuming.

\section{ACKNOWLEDGEMENTS}

Authors wish to acknowledge the financial support received from General Electric-Healthcare and Research Councils UK to carry out the study discussed in this paper.

\section{References:}

[1]. Birkmeyer JD, Sharp SM, Finlayson SRG, Fisher ES, Wenneber JE (1998) "Variation profiles of common surgical procedures” Surgery; 5: 917-923.

[2]. Vayda E. (1973), “A comparison of surgical rates in Canada and in England and Wales” N Engl J Med; 23: 1224-1236.

[3]. Groff JY, Mullen PD, Byrd T, Shelton AJ, Lees E, Goode J. (2000) "Decision making, beliefs, and attitudes toward hysterectomy: a focus group study with medically underserved women in Texas" $J$ Women's Health Gend Based Med; 9: 39-50.

[4]. Smith R. (1991) "Where is the wisdom? The poverty of medical evidence" Br Med J 1991; 303: 798-799.

[5]. Sanwlippo JS, Robinson CL. The Risk Management Handbook for Healthcare Professionals. London: The Partenon Publishing Group Ltd, 2002.

[6]. Weingart SN, Wilson RM, Gibberd RW, Harrison B. (2000) "Epidemiology of medical error" Br Med J; 320: 774-777.

[7]. Kitchiner D, Bundred PE. (1999) "Clinical pathways. A practical tool for specifying, evaluating and improving the quality of clinical practice" MJA; 170: 54-55.

[8]. Pearson SD, Goulart-Fisher D, Lee TH. (1995) "Critical pathways as a strategy for improving care: problems and potential” Ann Intern Med; 12: 941-948.

[9]. Archer SB, Burnett RJ, Flesch LV, et al., (1997) "Implementation of a clinical pathway decreases length of stay and hospital charges for patients undergoing total colectomy and ileal pouch/anal anastomosis" Surgery; 4: 699-705.

[10]. Wilson J. (1998) “Integrated care management” Br J Nurs; 4: 201-202.

[11]. Wentworth DA, Atkinson RP, (1996) "Implementation of an acute stroke program decreases hospitalisation costs and length of stay" Stroke;27: 1040-1043.

[12]. Willis B, Kim LT, Anthony T, Bergen PC, Nwariaku F, Turnage RH (2000) "A critical pathway for inguinal hernia repair reduces hospital admissions” J Surg Res; 1: 13-17.

[13]. Campbell, H., Hotchkiss, R., Bradshaw, N., Porteous, M. (1998) "Integrated care pathways" British Medical Journal; 316: 133-144.

[14]. Panella, M., Marchisio, S., Di Stanislao, F. (2003) "Reducing clinical variations with clinical pathways: do pathways work?” International Journal for Quality in Health Care; 15(6): 509-521

[15]. van Vliet, EJ, Sermeus, W., van Gaalen, CM, Sol, J C A, Vissers, JMH, (2010) "Efficacy and efficiency of a lean cataract pathway: a comparative study" Qual Saf Health Care; 19: 13.

[16]. Ould, M.A., (2005), “Business Process Management: A Rigorous Approach”, Meghan-Kiffer Press.

[17]. Nagesh Shukla, John E. Keast, Darek Ceglarek, (2014) Improved workflow modelling using role activity diagram-based modelling with application to a radiology service case study, Computer Methods and Programs in Biomedicine, Available online 5 June 2014, ISSN 0169-2607, http://dx.doi.org/10.1016/j.cmpb.2014.05.005

[18]. Wilde, K., (2009) "West Midlands Stroke Research Network Newsletter”, National Institute for Health Research

[19]. UK DoH, (2006) "Improving stroke services: a guide for commissioners" Product number: 278329, Gateway reference: 7418, Pages: 50, Copyright holder: Crown.

[20]. UK DoH, (2010) "Department of Health: Progress in improving stroke care”.

[21]. Crabbe JP, Frank CL, Nye WW (1994) "Improving report turnaround time: an integrated method using data from a radiology information system,” American Journal for Roentgenology; 163:1503-1507.

[22]. Staccini, P., Joubert, M., Quaranta, J.F., and Fieschi, M. (2005) "Mapping care processes within a hospital: from theory to a web-based proposal merging enterprise modelling and ISO normative principles” International Journal of Medical Informatics;74(2-4): 335-344

[23]. Mould, G, Bowers, J, Ghattas, M (2010) "The evolution of the pathway and its role in improving patient care” Qual Saf Health Care, 19: 14. 
[24]. Harbison, J, Hossain, O, Jenkinson, D, Davis, J, Louw, S. J., and Ford, G. A., (2003) "Diagnostic Accuracy of Stroke Referrals From Primary Care, Emergency Room Physicians, and Ambulance Staff Using the Face Arm Speech Test” Stroke; 34: 71-76.

[25]. Mohd Nor, A., Davis, J., Sen, B., Shipsey, D., Louw, S. J, Dyker, A. G., Davis, M., Ford, G. A., (2005) "The Recognition of Stroke in the Emergency Room (ROSIER) scale: development and validation of a stroke recognition instrument” The Lancet Neurology;4(11): 727 - 734.

[26]. Kasner, S. E., Chalela, J. A., Luciano, J. M., Cucchiara, B. L., Raps, E. C., McGarvey, M. L., Conroy, M. B., and Localio A. R., (1999) "Reliability and Validity of Estimating the NIH Stroke Scale Score from Medical Records” Stroke; 30:1534-1537

[27]. Kobayashi, A., Wardlaw, J. M., Lindley, R. I., Lewis, S. C., Sandercock, P. A.G., Czlonkowska, A., (2009) "Oxfordshire Community Stroke Project Clinical Stroke Syndrome and Appearances of Tissue and Vascular Lesions on Pretreatment CT in Hyperacute Ischemic Stroke Among the First 510 Patients in the Third International Stroke Trial (IST-3)” Stroke; 40: 743-748.

[28]. Bayer, S., Petsoulas, C., Cox, B., Honeyman, A. and Barlow, J., (2010) "Facilitating stroke care planning through simulation modelling” Health Informatics Journal; 16(2): 129-143.

[29]. Dodds S., (2005) “Designing improved healthcare processes using discrete event simulation” $\mathrm{Br} J$ Healthcare Comput Inf Manage; 22 (5): 14-16.

[30]. Connelly LG, Bair AE. (2004) "Discrete event simulation of emergency department activity: a platform for system-level operations research" Academy of Emergency Medicine; 11: 1177-84.

[31]. Kotiadis K, Mackenzie M. (2004) “Simulation modelling for intermediate care" $\mathrm{Br} J$ Healthcare Manage;10 (8): 240-6.

[32]. Stevens, W., Myers, G., Constantine, L. (1974) "Structured Design", IBM Systems Journal;13(2),:115139.

[33]. Rother, M., Shook, J. (2003), “Learning to See - Value-stream Mapping to Create Value and Eliminate Muda” Lean Enterprise Institute, ISBN: 9780966784305.

[34]. Patel, N. V. (2000) "Healthcare Modelling through Role Activity Diagrams for Process-Based Information Systems Development,” Requirement Engineering; 5(2): Pages: 83-92.

[35]. Wardlaw, J. M., Seymour, J., Cairns, J., Keir, S., Lewis, S., and Sandercock, P., (2004) "Immediate Computed Tomography Scanning of Acute Stroke Is Cost-Effective and Improves Quality of Life" Stroke; 35: 2477-2483

[36]. Chalela JA, Kidwell CS, Nentwich LM, Luby M, Butman JA, Demchuk AM, Hill MD, Patronas N, Latour L, Warach S. (2007), Magnetic resonance imaging and computed tomography in emergency assessment of patients with suspected acute stroke: a prospective comparison, Lancet; 369 (9558): 293-8.

[37]. Duguay, C., Chetouane, F. (2007) “Modelling and Improving Emergency Department Systems using Discrete Event Simulation” Simulation; 83(4) : 311-320.

[38]. Molema, J J W, Groothuis, S., Baars, I J, Kleinschiphorst, M., Leers, E.G.E, Hasman, A., van Merode, G. G., (2007), "Healthcare System design and Parttime working doctors” Health Care Management Science; 10: 365-371

[39]. VanBerkel, P. T., and Blake, J. T., (2007) “A comprehensive simulation for wait time reduction and capacity planning applied in general surgery” Health Care Management Science;10: 373-385.

[40]. Davies R, Roderick P, Crabbe D, Raftery P, Patel P, Goddard JR. (2002) "A simulation to evaluate screening for helicobacter pylori infection in the prevention of peptic ulcers and gastric cancers" Health Care Manage Sci: 249-58.

[41]. Brailsford S, Rauner M, Gutjahr E, Zeppelzauer W, (2006) “Combined discrete-event simulation and ant colony optimisation approach for selecting optimal screening policies for diabetic retinopathy" Computational Manage Sci;4: 59-83.

[42]. NAO, (2005) "Reducing Brain Damage: Faster access to better stroke care” HC: 452 2005-2006, ISBN: 010293570X

[43]. Adamson, J, Beswick, A. and Ebrahim, S. (2004). “Stroke and Disability” Journal of Stroke and Cerebrovascular Diseases;13: 171-177.

[44]. Nagesh Shukla, John Keast, Darek Ceglarek, (2014) Modelling variations in hospital service delivery based on real time locating information, Applied Mathematical Modelling; 38(3) :878-893

[45]. Stroke Unit Trialists' Collaboration (1997) "Collaborative systematic review of the randomised trials of organised inpatient (stroke unit) care after stroke" BMJ; 314(7088): 1151-1159.

[46]. Sarrechia M, Van Gerven E, Hermans L, Deneckere S, Sermeus W, Panella M, Spitz B, Vanhaecht K. (2013) "Variation in 17 obstetric care pathways: potential danger for health professionals and patient safety?” J Adv Nurs;69(2):278-85

[47]. Ryan, J., Heavey, C., (2006) "Process modeling for simulation" Computers in Industry; 57(5): 437 - 450. 
[48]. West, D. 2010. Hospital bed transfers put thousands of patients at risk of infection. Nursingtimes.net [Online]. Accessed Feb 2, 2015, Available: http://www.nursingtimes.net/specialist-news/infectioncontrol-news/hospital-bed-transfers-put-thousands-of-patients-at-risk-of-infection/5019997.article

[49]. Jonathan Karnon, James Stahl, Alan Brennan, J. Jaime Caro, Javier Mar, Jörgen Möller, (2012), "Modeling Using Discrete Event Simulation: A Report of the ISPOR-SMDM Modeling Good Research Practices Task Force-4" Med Decis Making; 32(5): 701-711

[50]. Nagesh Shukla, Rohan Wickramasuriya, Andrew Miller, Pascal Perez, An approach to plan and evaluate the location of radiotherapy services and its application in the New South Wales, Australia, Computer Methods and Programs in Biomedicine, Available online 13 August 2015, ISSN 0169-2607, DOI: http://dx.doi.org/10.1016/j.cmpb.2015.08.005.

[51]. Sangi M, Win KT, Shirvani F, Namazi-Rad M-R, Shukla N (2015) Applying a Novel Combination of Techniques to Develop a Predictive Model for Diabetes Complications. PLoS ONE 10(4): e0121569. doi:10.1371/journal.pone.0121569 


\section{List of Figures}

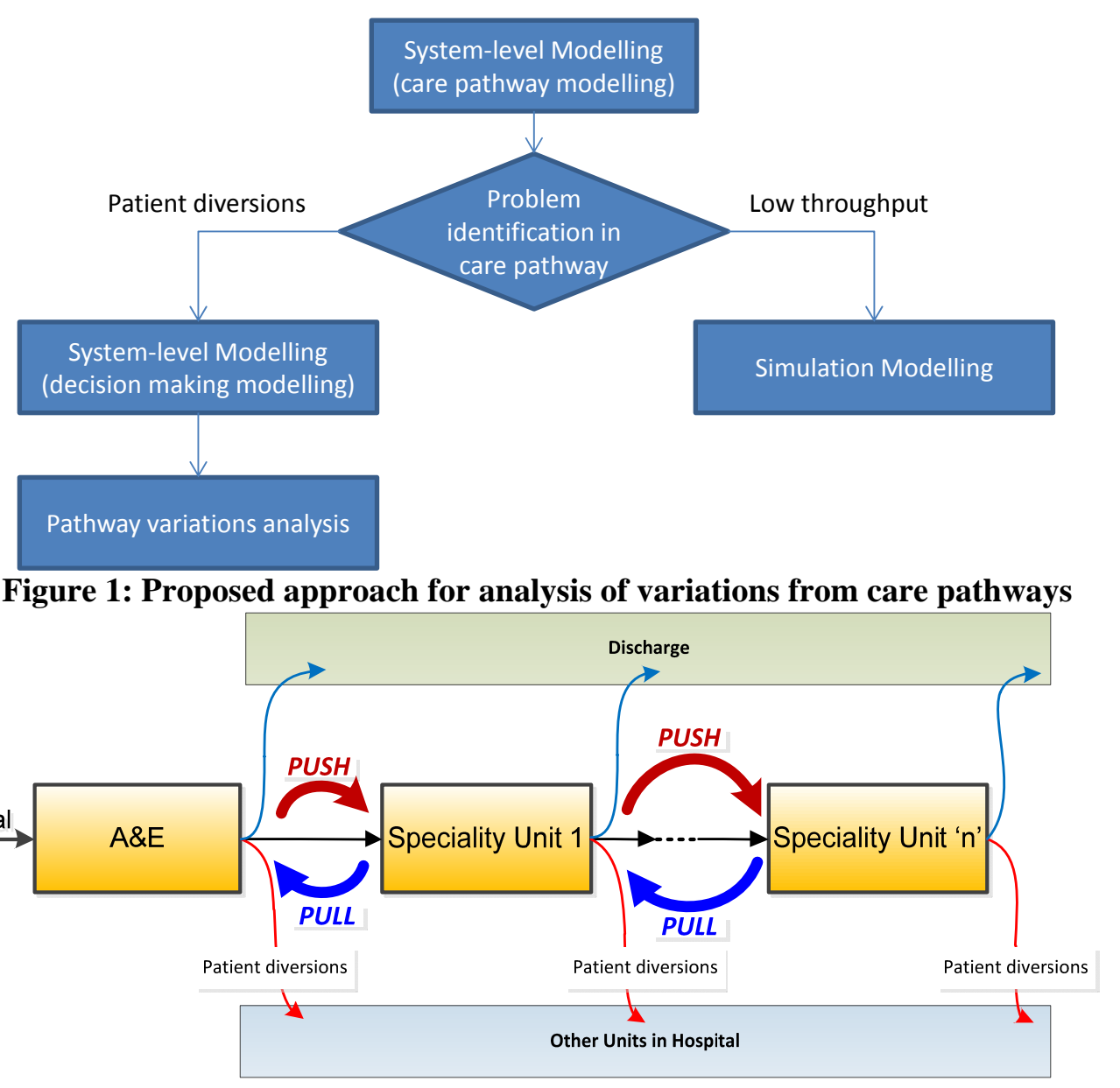

Figure 2: Push and Pull factors between speciality units involved in patient care pathway

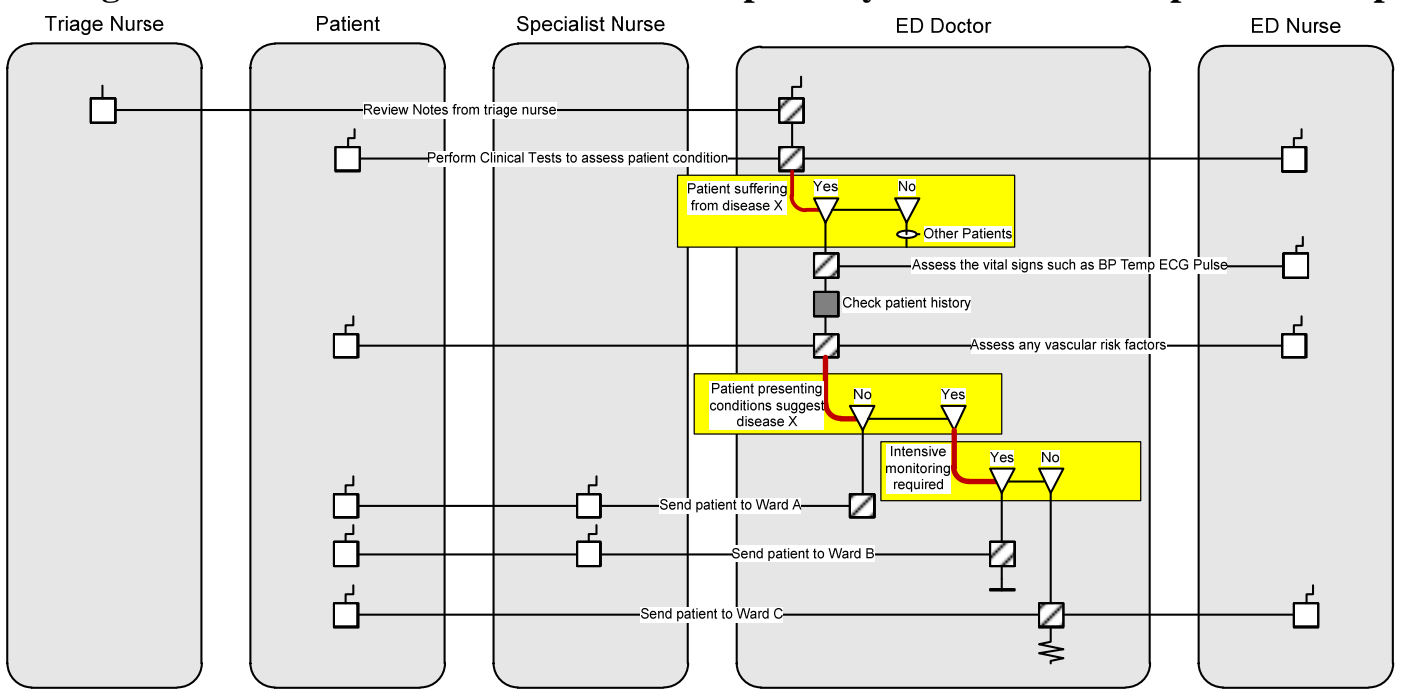

Figure 3: RAD of a process illustrating decisions or case refinements included as $C R$ 


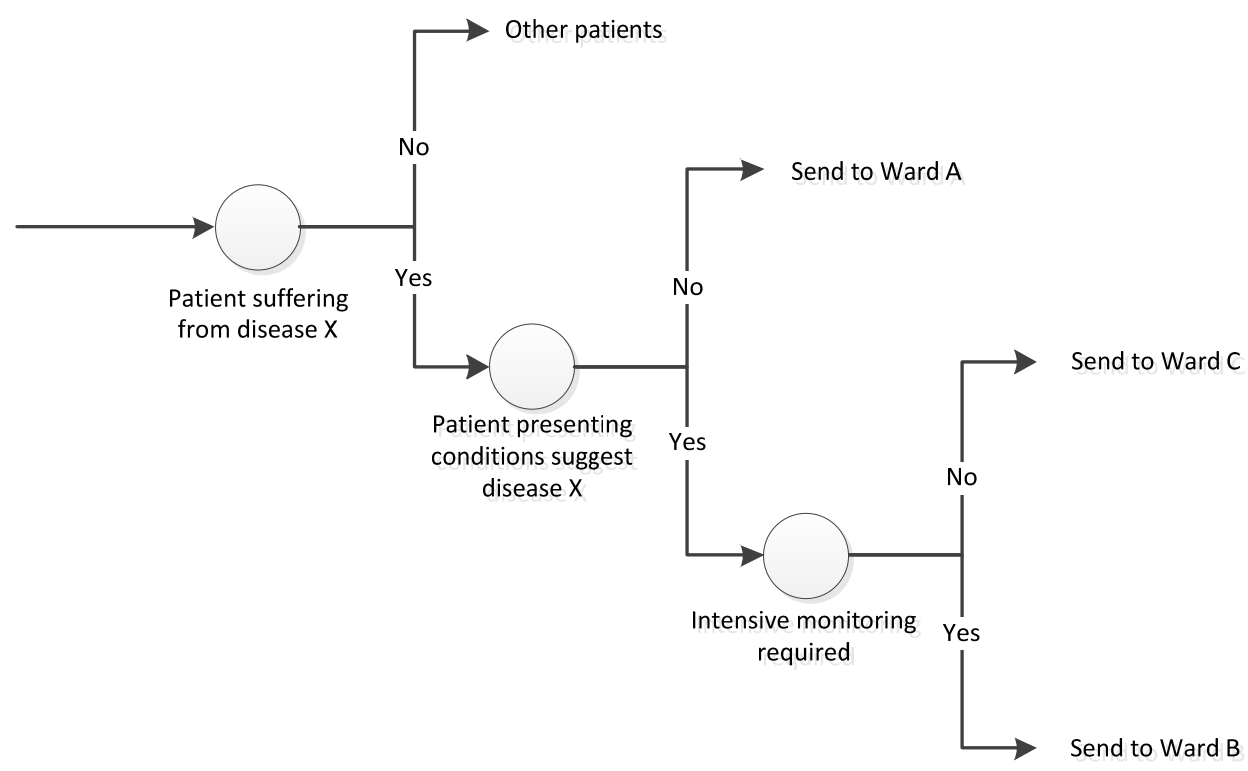

Figure 4: Conceptual representation of $C R$ represented in RAD

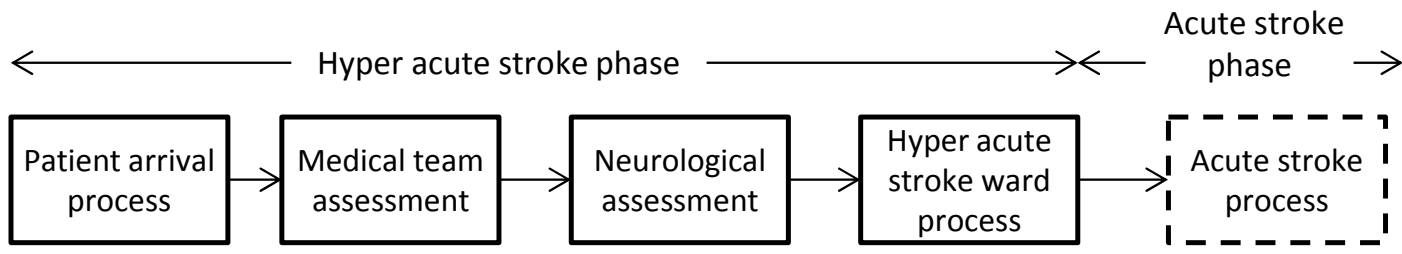

Figure 5: Processes involved in stroke care pathway of a hospital

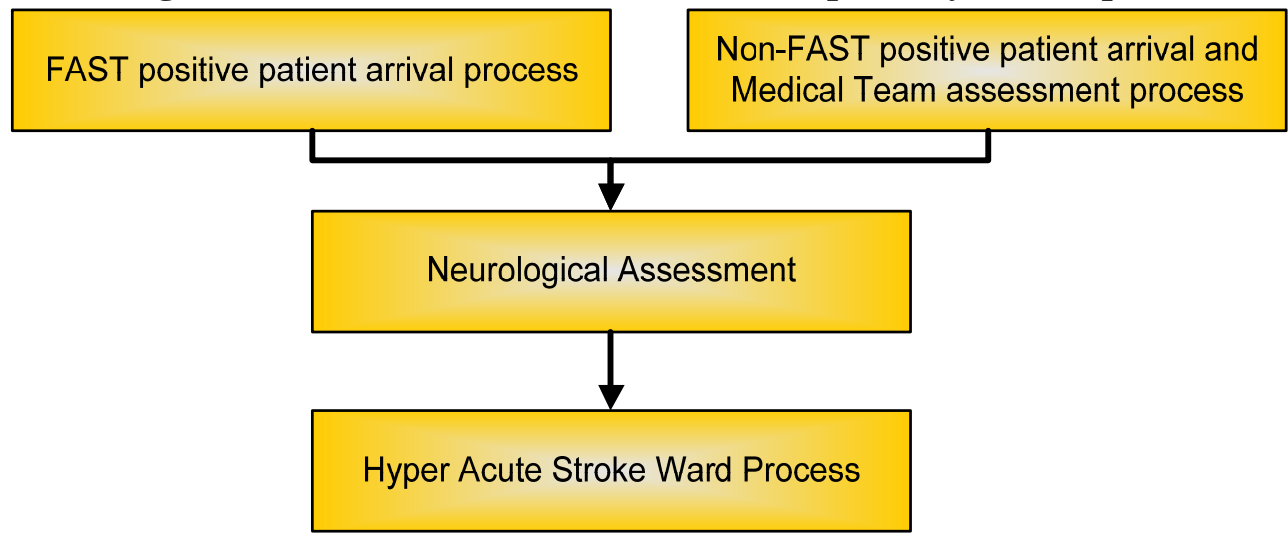

Figure 6: Sub-processes of hyper acute phase of stroke care pathway 


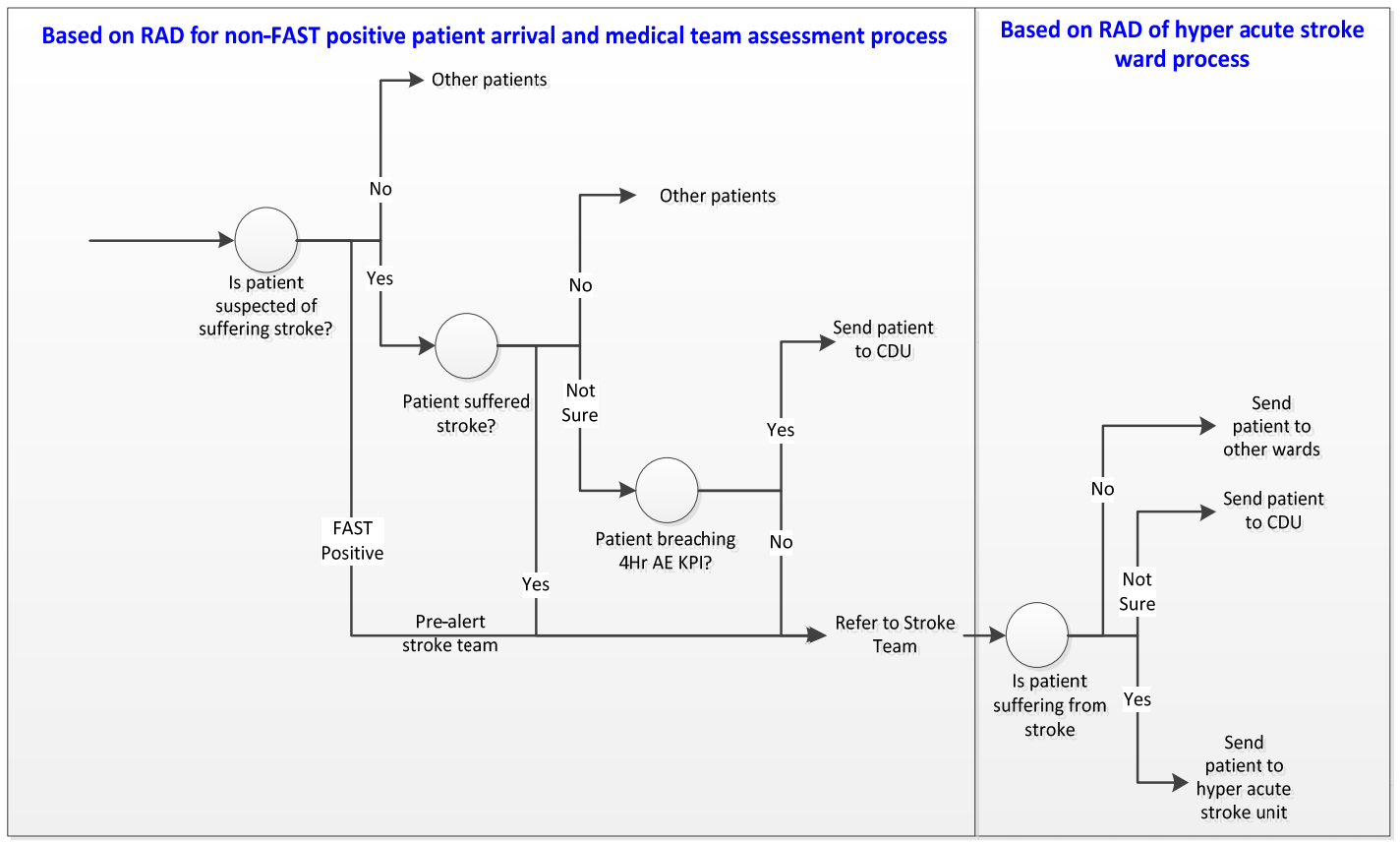

Figure 7: Conceptual diagram for the case refinements (CR) represented in RADs 


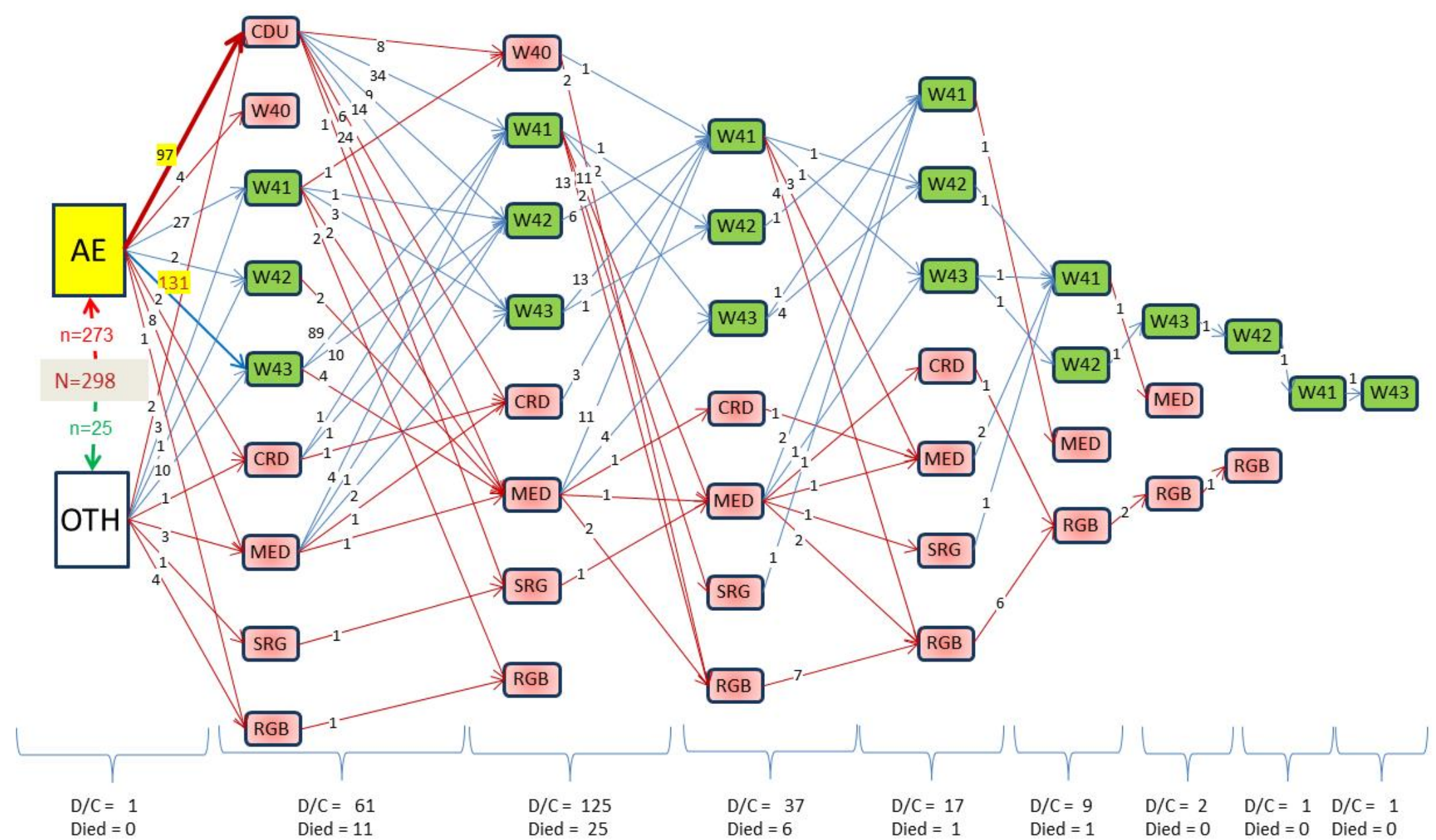

Figure 8: Patient diversion from stroke care pathways based on hospital data (April 2010 - August 2010), OTH: other sources, D/C: patient discharged 


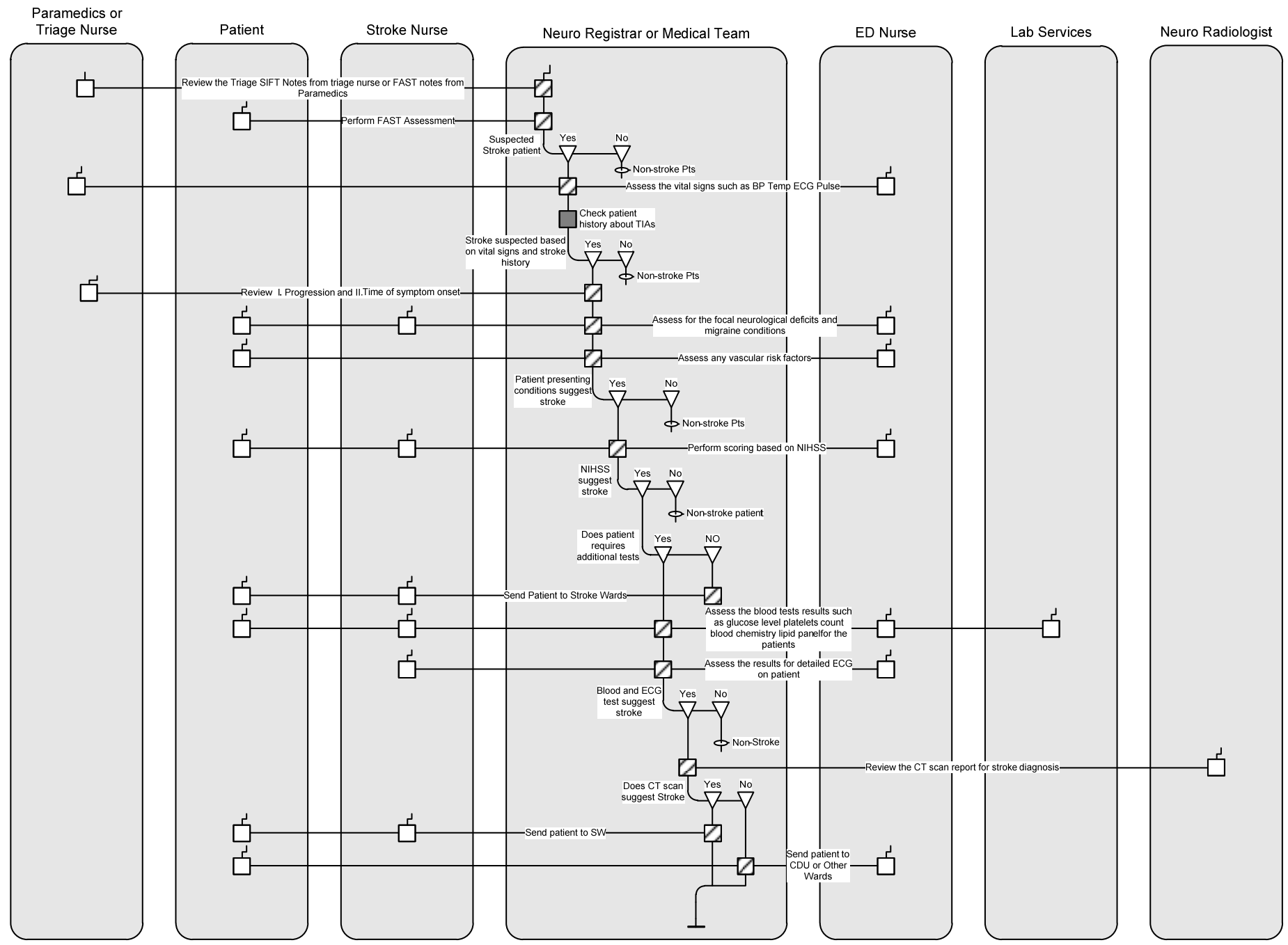

Figure 9: RAD of decision making process for identifying stroke; (see Table A.1 for RAD notations) 


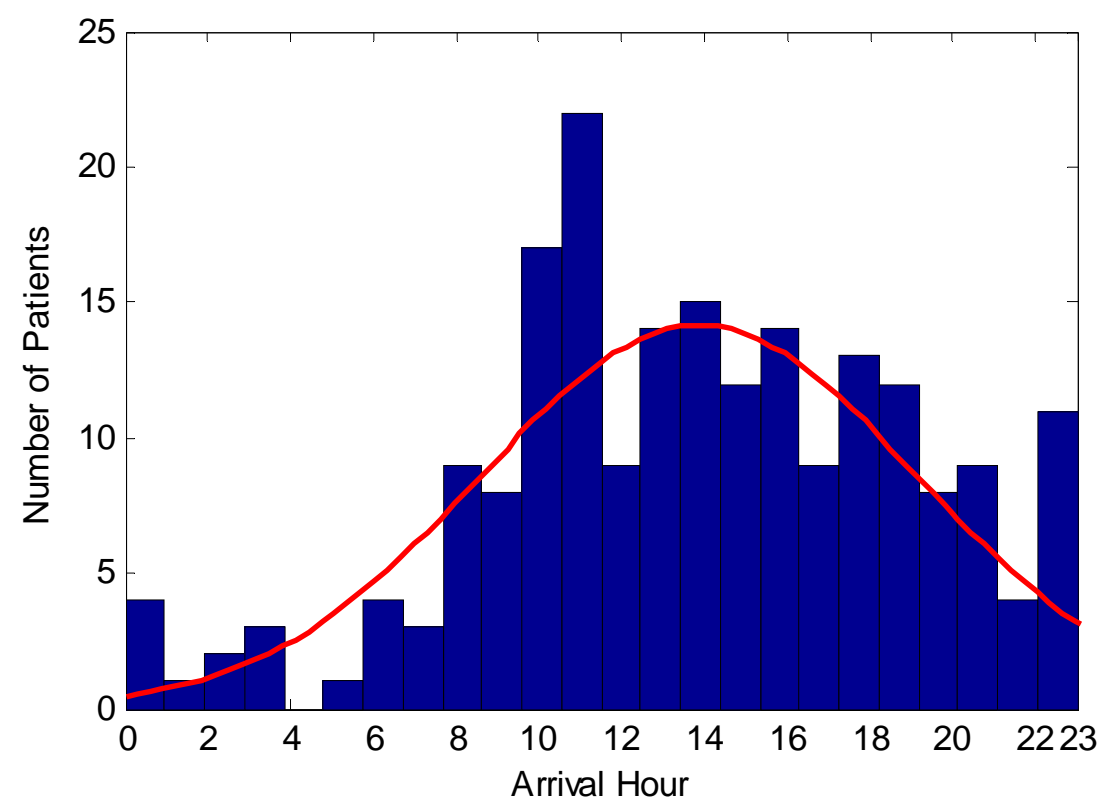

Figure 10: Patient arrival time distributions
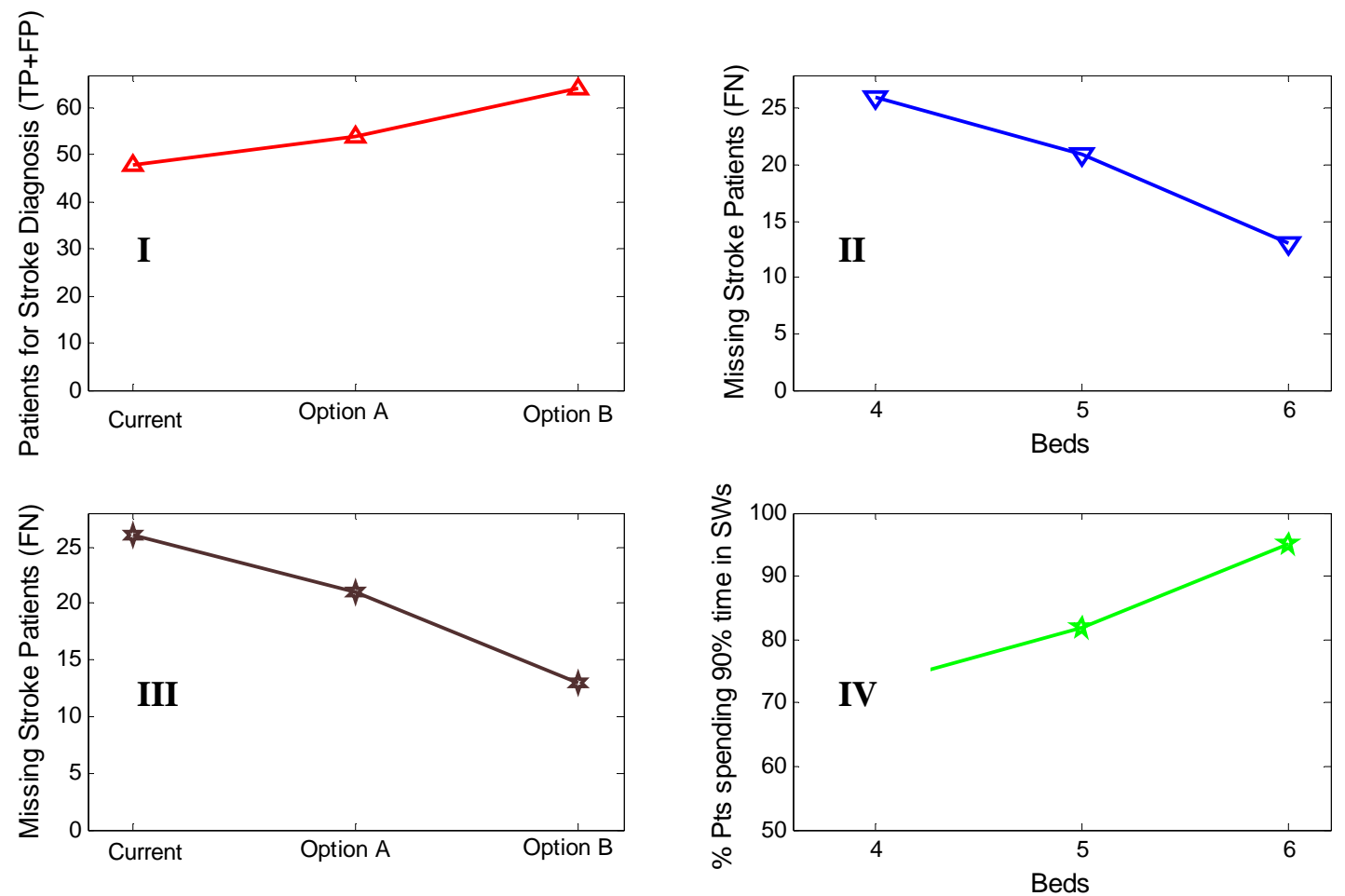

Figure 11: Simulation analysis for Current, Option A, and Option B

\section{List of Tables}

Table 1: Average percentages of patients of each $\boldsymbol{k} \in \boldsymbol{P} \boldsymbol{t}_{\text {type }}$

\begin{tabular}{|c|c|}
\hline Patient Type $\left(\right.$ Pt $\left._{\text {type }}\right)$ & Percentages \\
\hline 1 & 24.5 \\
\hline
\end{tabular}




\begin{tabular}{|l|l|}
\hline 2 & 32.2 \\
\hline 3 & 07.7 \\
\hline 4 & 05.6 \\
\hline 5 & 30.0 \\
\hline
\end{tabular}

Table 2: Performance of stoke care centre in a hospital due to implementation of Option B

\begin{tabular}{|c|c|c|}
\hline \multicolumn{2}{|c|}{$\begin{array}{c}\text { Time } \\
\text { (Annual \& Quarter) }\end{array}$} & $\begin{array}{c}\text { Stroke Care } \\
\text { KPI (80/90) }\end{array}$ \\
\hline \multicolumn{2}{|c|}{2011 - Q1 } & $\mathbf{8 4 . 9 7}$ \\
\hline \multirow{2}{*}{2010} & Q4 & $\mathbf{8 4 . 2 4}$ \\
\cline { 2 - 3 } & Q3 & 77.12 \\
\cline { 2 - 3 } & Q2 & 75.66 \\
\cline { 2 - 3 } & Q1 & 73.00 \\
\hline \multicolumn{2}{|c|}{$2009 / 10^{*}$} & 62.20 \\
\hline \multicolumn{2}{|c|}{$2008 / 09 *$} & 56.76 \\
\hline
\end{tabular}




\section{Appendix A}

\section{RAD based care pathway modelling:}

The list of roles involved in the hyper acute phase of stroke care pathway is elicited by interviewing the stroke services manager in hospital, who manages and administers the stroke pathway within hospital. The key roles involved within the hyper acute phase are Paramedics, A\&E Receptionist, Resus Nurse, Emergency Nurse, Triage Nurse, Stroke Nurse, Neurology Registrar, CT Radiographer, Radiologist, Medical Team in A\&E (Consultant/Senior Health Officer (SHO)/Specialist Registrar $(\mathrm{SpR})$ ), and Modern Matron are identified. Member of staff performing each roles were interviewed regarding details of their involvement in the stroke pathway from start to end. The interviews were recorded using the digital recorder DS-40 from Olympus. After recording, audio files were transcribed into Microsoft Word (2007). These textual transcripts are used to build RAD of the hyper acute phase of stroke care pathway based on RAD modelling steps (see Section 2.1.1). Notations for RAD symbols are illustrated in Table A.1.

Table A. 1: Notations for RAD symbols

\begin{tabular}{|c|c|c|c|c|c|}
\hline $\begin{array}{c}\text { RAD } \\
\text { concept }\end{array}$ & Examples & $\begin{array}{l}\text { Graphical } \\
\text { notations }\end{array}$ & $\begin{array}{c}\text { RAD } \\
\text { concep } \\
t\end{array}$ & Examples & $\begin{array}{c}\text { Graphical } \\
\text { notations }\end{array}$ \\
\hline Role & $\begin{array}{l}\text { Clinical doctors, } \\
\text { porters, nurses, } \\
\text { technicians }\end{array}$ & & Activity & $\begin{array}{l}\text { Move patient to } \\
\text { changing room, } \\
\text { position patient } \\
\text { on table }\end{array}$ & \\
\hline $\begin{array}{c}\text { Interactio } \\
n\end{array}$ & $\begin{array}{l}\text { Pass } X \text {-ray of the } \\
\text { patient from } \\
\text { technician to } \\
\text { radiology doctors }\end{array}$ & & $\begin{array}{l}\text { Case } \\
\text { refinem } \\
\text { ent }\end{array}$ & $\begin{array}{c}\text { Decision } \\
\text { question: Does } \\
\text { patient require } \\
\text { contrast } \\
\text { injection? } \\
\text { Outcome: Yes, } \\
\text { or No. } \\
\end{array}$ & $\varphi-\nabla$ \\
\hline $\begin{array}{c}\text { Part } \\
\text { refinemen } \\
t\end{array}$ & $\begin{array}{l}\text { Patient booking, } \\
\text { and printing } \\
\text { patient non } \\
\text { attendance letters }\end{array}$ & $\triangle \triangle \triangle \triangle$ & Trigger & $\begin{array}{l}\text { Arrival of } \\
\text { patient scan } \\
\text { request }\end{array}$ & \\
\hline State & $\begin{array}{l}\text { Scan is finished, } \\
\text { patient can have } \\
\text { scan }\end{array}$ & Q & Loop & $\begin{array}{l}\text { Multiple attempt } \\
\text { to give injection }\end{array}$ & \\
\hline $\begin{array}{c}\text { Replicatio } \\
n\end{array}$ & $\begin{array}{l}\text { Maximum two } \\
\text { repeats in giving } \\
\text { injections }\end{array}$ & * & $\begin{array}{l}\text { Encaps } \\
\text { ulated } \\
\text { process }\end{array}$ & $\begin{array}{l}\text { Perform patient } \\
\text { scanning }\end{array}$ & 向 \\
\hline Start role & $\begin{array}{l}\text { Patient scan } \\
\text { request vetting by } \\
\text { radiology doctors }\end{array}$ & 向 & $\begin{array}{l}\text { Other } \\
\text { work }\end{array}$ & & $\sum$ \\
\hline Stop & & 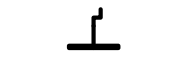 & & & \\
\hline
\end{tabular}

\section{Stroke care pathway process based on RADs:}

The stroke care pathway starts with the FAST positive patient arrival at A\&E (see Fig. A.1 related to FAST positive patients). The process starts by a trigger representing paramedic staff arrival at patient location and performing FAST assessment. If patient is found FAST positive, a FAST positive alert is sent to resus nurse in A\&E by paramedics control room. Resus nurse then alerts acute stroke team comprising of stroke nurse (in hyper acute stroke ward), neurology registrar, and emergency department (ED) specialist registrar (SpR). Stroke nurse from the hyper acute stroke ward report to the A\&E resus area based on the pre-alert and will wait for patient arrival. 
Simultaneously, paramedics transport patient to $A \& E$ and hands over the patient to resus nurse and stroke nurse. Further, paramedics hands over the ambulance log sheet (paramedics form) to the A\&E receptionist, who registers patient demographics and medical condition into various IT systems. FAST positive patients are then normalized and initial tests are performed by resus nurse together with stroke nurse in resus area followed by neurologic assessments.

Figure A.2 illustrates the process of non-FAST patient arrival and medical team assessment at A\&E. This process starts when patients are identified as non- FAST positive by paramedics. These patients are then transported to the local $A \& E$ and handovers to A\&E receptionist, who registers patient into IT systems and finally notifies the triage nurse of patient arrival and start the triage assessment. Initial patient assessment is performed by the triage nurse and decision is made about patient having suffered stroke. If patient is FAST positive then resus nurse is alerted and FAST positive patient process is initiated.

However, if patient is not FAST positive and triage nurse suspect stroke, then medical team in A\&E is notified with the help of causality card which details about the patient conditions in triage. Then medical team (comprising of $\mathrm{SHO} / \mathrm{SpR} /$ Consultant) will assess the patient conditions and if patient conditions are unclear will order blood test, electrocardiogram (ECG) test, computed tomography (CT) scan of the patient. Medical team will send these patients to clinical decisions unit (CDU) if patient is nearing 4 hours of A\&E KPI for further assessment based on tests. CDU is a ward used for the patients who has complex presenting conditions and requires final decision about their diagnosis. It acts as a buffer to the A\&E for the patients breaching 4 hour KPI of A\&E. Then medical team makes decision about the patient diagnosis after receiving the test reports. If patient is identified to have suffered stroke then the neurology registrar and stroke nurse is notified about the stroke patient who is followed by the neurologic assessment.

Figure A.3 illustrates the neurological assessment process for the stroke patients. Stroke nurse assess the initial patient condition and if conditions are complicated then neurology registrar are called to assess patient otherwise neurology registrar supervises stroke nurse by means of telephone. Then CT scanning is ordered and performed by stroke nurse and CT radiographer. Finally, patient is out of the CT scanner and the images are interpreted and reported by the neuro-radiologist.

Figure A.4 illustrates the hyper acute stroke ward process which is initiated after the neurological assessment of stroke patients. Stroke nurse performs ECG, and blood test in collaboration with ECG technician and Lab services. Reports of these tests together with the assessment of neurology registrar are used to make decision about if a patient has suffered stroke. If patient is identified to be stroke patients, then they are sent to hyper acute stroke wards and thrombolysis (tPA) eligibility is assessed is provided to stroke patients. 


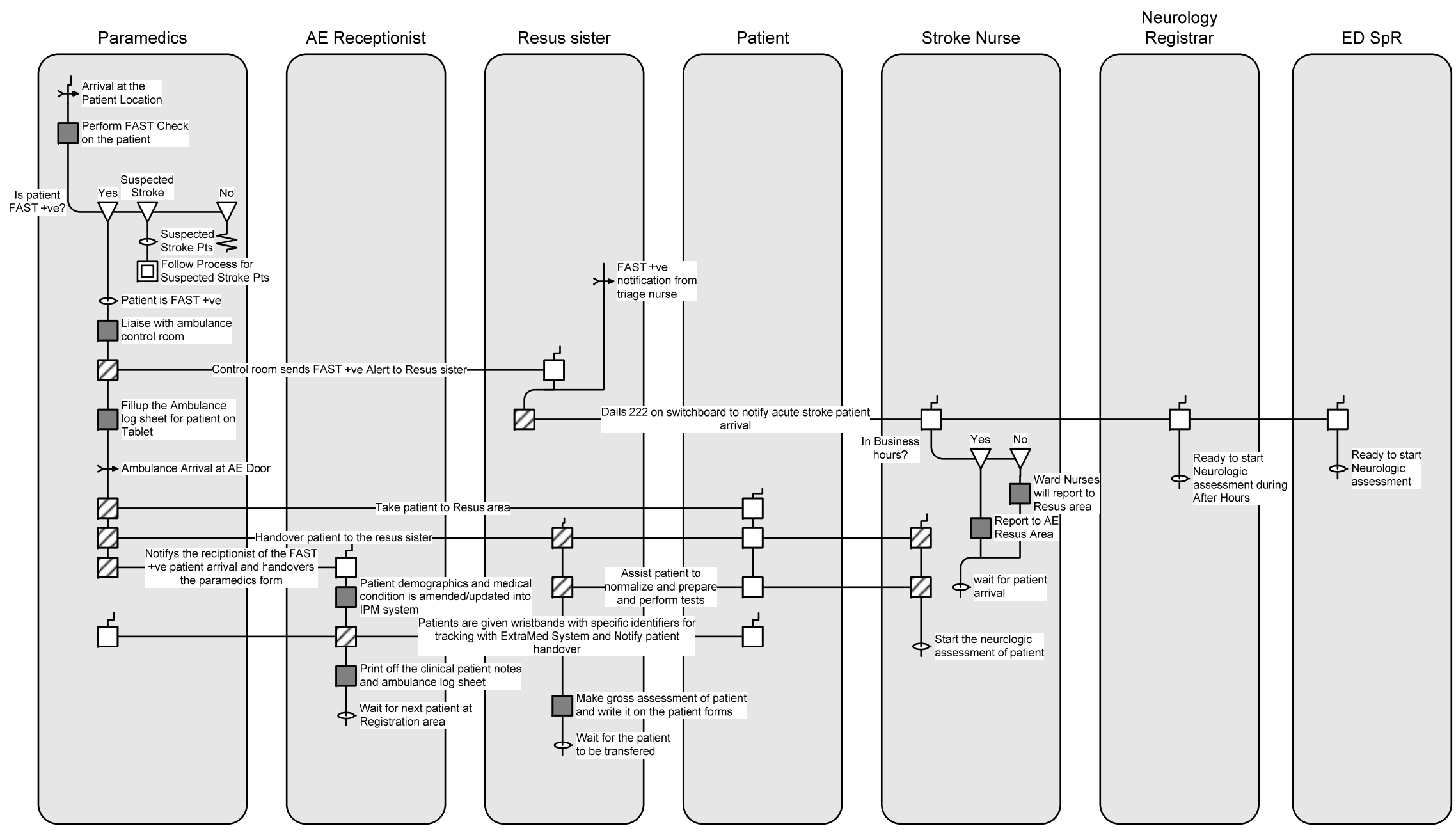

Figure A.1: RAD for FAST positive patient arrival process 


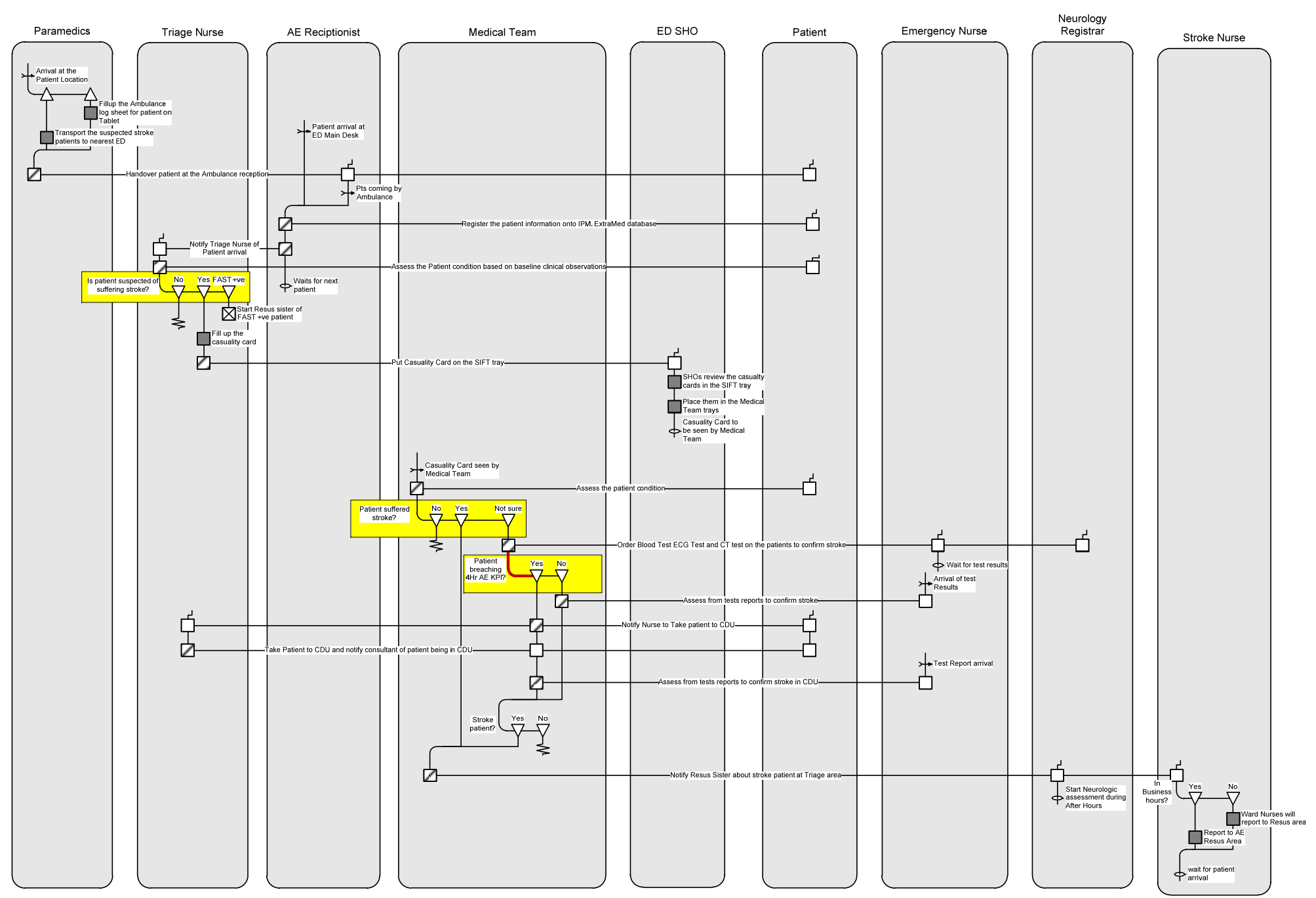

Figure A.2: RAD of non-FAST positive patient arrival and medical team assessment process 


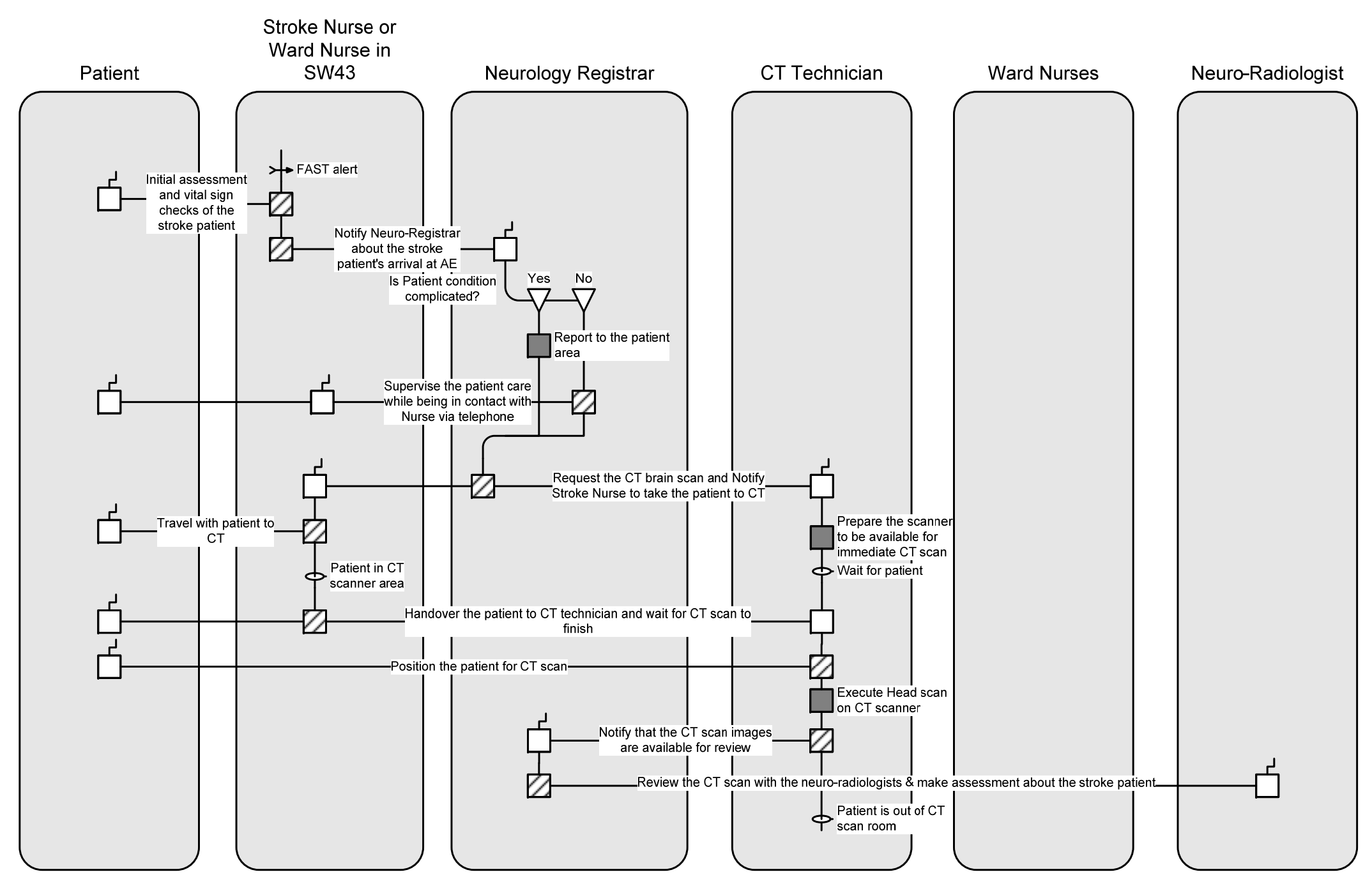

Figure A.3: RAD of neurological assessment process of stroke patients 


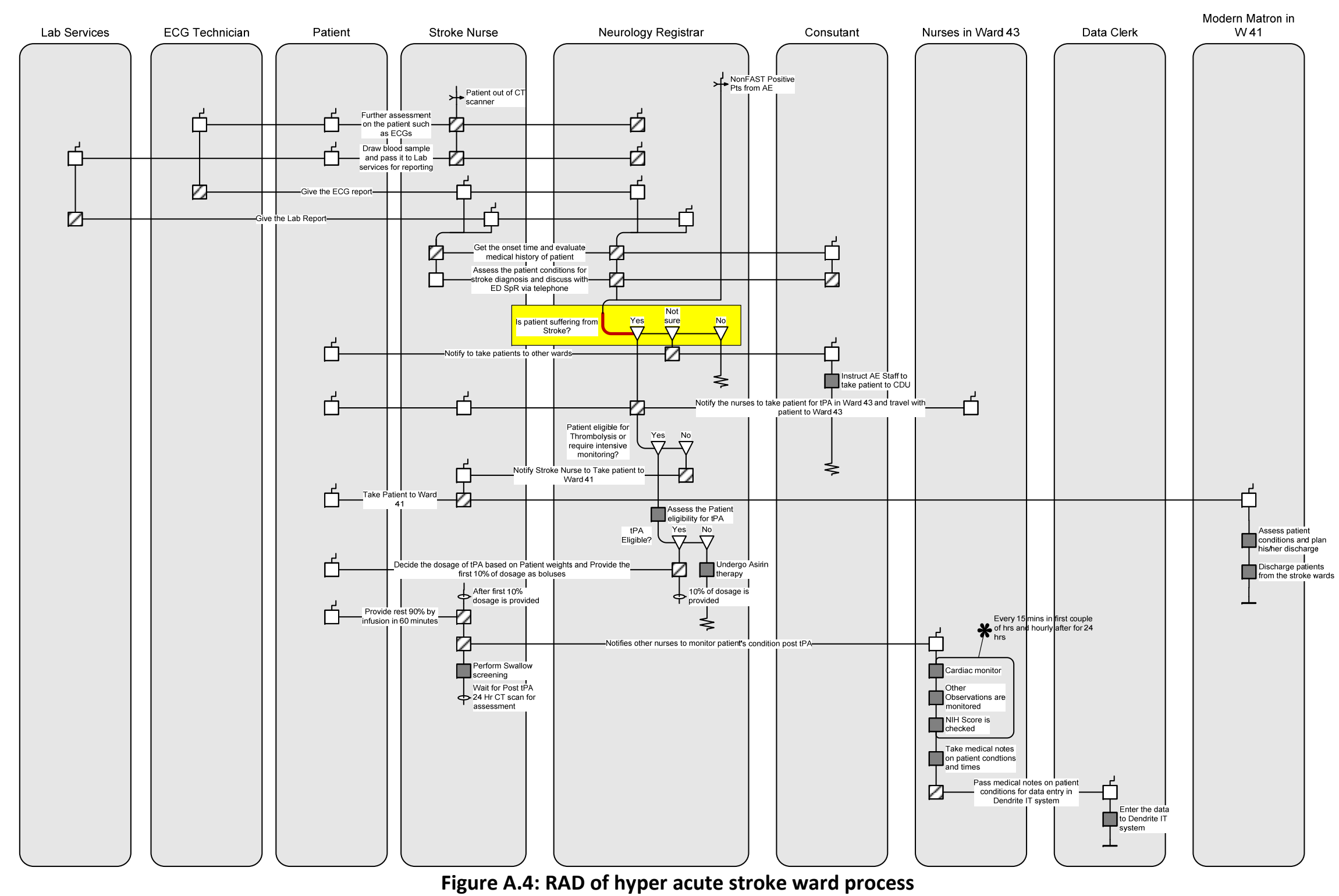

